\title{
Redox-sensitive micelles composed of disulfide- linked Pluronic-linoleic acid for enhanced anticancer efficiency of brusatol
}

This article was published in the following Dove Press journal:

International Journal of Nanomedicine

\author{
Jinming Zhangl,* \\ Xiaobin Fangl,* \\ Zeyong $\mathrm{Li}^{2}$ \\ Hon Fai Chan ${ }^{3}$ \\ Zhixiu Lin ${ }^{4}$ \\ Yitao Wang' \\ Meiwan Chen'
}

'State Key Laboratory of Quality Research in Chinese Medicine, Institute of Chinese Medical Sciences, University of Macau, Macau, China; ${ }^{2}$ Department of Laboratory Medicine, Guangdong No 2 Provincial People's Hospital, Guangzhou, China; ${ }^{3}$ Department of Biomedical Engineering, Columbia University, New York, NY, USA; ${ }^{4}$ Faculty of Science, School of Chinese Medicine, Chinese University of Hong Kong, Shatin, Hong Kong, China

*These authors contributed equally to this work
Correspondence: Meiwan Chen Institute of Chinese Medical Sciences, University of Macau, Av Padre Tomas Pereira S.J., Taipa, Macau, China Email mwchen@umac.mo

Zeyong Li

Department of Laboratory Medicine, Guangdong No 2 Provincial People's Hospital, Nol, Shiliugang Road, Haizhu District, Guangzhou 510317, China Email sunnar78@I26.com

\begin{abstract}
Brusatol (Bru) exhibits promising anticancer effects, with both proliferation inhibition and chemoresistance amelioration activity. However, the poor solubility and insufficient intracellular delivery of Bru greatly restrict its application. Herein, to simultaneously utilize the advantages of Pluronics as drug carriers and tumor microenvironment-responsive drug release profiles, a flexible amphiphilic copolymer with a polymer skeleton, that is, Pluronic ${ }^{\circledR}$ F68 grafting with linoleic acid moieties by redox-reducible disulfide bonds (F68-SS-LA), was synthesized. After characterization by ${ }^{1} \mathrm{H}$-nuclear magnetic resonance and Fourier transform infrared spectroscopy, the redox-sensitive F68-SS-LA micelles were self-assembled in a much lower critical micelle concentration than that of the unmodified F68 copolymer. Bru was loaded in micelles (Bru/SS-M) with high loading efficiency, narrow size distribution, and excellent storage stability. The redox-sensitive Bru/SS-M exhibited rapid particle dissociation and drug release in response to a redox environment. Based on the enhanced cellular internalization, Bru/SS-M achieved higher cytotoxicity in both Bel-7402 and MCF-7 cells compared with free Bru and nonreducible micelles. The improved anticancer effect was attributed to the remarkably decreased mitochondrial membrane potential and increased reactive oxygen species level as well as apoptotic rate. These results demonstrated that F68-SS-LA micelles possess great potential as an efficient delivery vehicle for Bru to promote its anticancer efficiency via an oxidation pathway.
\end{abstract}

Keywords: poloxamer188, tumor micro-environment, glutathione, Brucea javanica, cancer treatment

\section{Introduction}

In view of the continually rising morbidity and mortality rates caused by cancer worldwide, it is imperative to develop effective therapeutic options. Unfortunately, chemotherapy, one of the most common cancer treatment approaches, ${ }^{1}$ is hampered by unsatisfactory therapeutic outcomes. The challenges of chemotherapy include the narrow therapeutic window of anticancer drugs, poor water solubility, short circulating half-life, insufficient tumor accumulation and non-selective delivery to tumor sites. ${ }^{2,3}$ Recent advances in nanoscale drug delivery (particle size: 20-250 nm) have drastically improved chemotherapy outcomes. ${ }^{4}$ Particularly, tumor-targeted nanocarriers have shown promising results in terms of higher intra-tumoral drug concentrations and lower normal tissue concentrations than free drug counterparts via an enhanced permeability and retention (EPR) effect or overexpressed tumor receptor binding. ${ }^{5-7}$ However, the therapeutic efficiency of nanomedicine in clinics remains questionable as a consequence of the excessively gradual drug release from nanovehicles. ${ }^{8-10}$ Redox-sensitive micelles have recently received increasing interest to facilitate drug 
bursts at the tumor site in response to the higher glutathione (GSH) concentrations in cancer cells $(\sim 10 \mathrm{mM})$ compared with $\sim 20 \mu \mathrm{M}$ GSH in normal physiological environments. ${ }^{11-14}$ This unique intracellular redox potential enables the versatile design of redox-sensitive micelles with improved intracellular drug delivery and enhances therapeutic efficiency.

Pluronic, a triblock copolymer comprising hydrophilic poly(ethylene oxide) (PEO) and lipophilic poly(propylene oxide) (PPO), has been widely used in nanodrug delivery systems with good biocompatibility and low toxicity. ${ }^{15-17}$ Based on its amphiphilic nature, single component Pluronic copolymer micelles can be formulated based on its hydrophobic PPO core and hydrophilic PEO corona. ${ }^{18}$ Pluronic F68, with a monomer unit ratio of 78/30/78, is approved for intravenous injection by the US Food and Drug Administration. Nevertheless, due to its high $\mathrm{PEO} / \mathrm{PPO}$ ratio, the drug encapsulation efficiency (EE) and stability of single F68 micelles are poor. ${ }^{19}$ Some strategies have been explored to enhance the kinetic stability of F68 micelles in the blood stream upon dilution and to reduce the critical micelle concentration (CMC), including generating mixed micelles with different Pluronic copolymers, ${ }^{20}$ F68-drug conjugates, ${ }^{21}$ and F68 complexed with other amphiphilic copolymers. ${ }^{22}$ However, both the copolymer complexes and chemical conjugation prodrug approaches require excess amounts of polymers, resulting in reduced drug loading (DL) efficiency. Reinforcing the hydrophobic core of F68 by conjugating additional lipophilic chains could effectively enhance the drug-encapsulation capacity and strengthen its stability.

One of the most prominent strategies in cancer chemoprevention is to regulate oxidative stress in both tumor development and response to anticancer therapies. ${ }^{23}$ Brusatol (Bru), a quassinoid isolated from the Chinese herbal medicine Brucea javanica fruit, ${ }^{24}$ demonstrates various bioactivities, including anticancer. ${ }^{25}$ Moreover, Bru can improve chemotherapy efficiency by inhibiting the up-expression of nuclear factor erythroid 2-related factor $2,{ }^{26}$ which contributes to chemoresistance. ${ }^{27,28}$ However, like numerous other anticancer candidates, its high hydrophobicity caused by the multiple hydrophobic rings in the molecular structure greatly limits clinical application through intravenous administration.

In this study, water-soluble amphiphilic F68 was grafted with linoleic acid (LA), ${ }^{29}$ an essential fatty acid with a chain length of 18 carbon atoms, mediated by the disulfide linkage to encapsulate Bru (Bru/F68-SS-LA). This approach aimed to decrease the $\mathrm{CMC}$ value, enhance the DL, and generate more substantial redox-sensitive micelles by self-assembly. More importantly, LA can be incorporated into the lipid bilayer of cells seamlessly, ${ }^{30}$ which results in higher cellular uptake of F68-SS-LA micelles. The physicochemical properties, such as the CMC, stability, micelle size, DL, and in vitro redox-responsive drug-release behavior were investigated. Furthermore, the enhanced cellular uptake, cytotoxicity, and related antioxidative pathways were examined in detail.

\section{Materials and methods Materials}

Poloxamer 188 (Pluronic F68, Mw 8350) was purchased from BASF Co. (Ludwigshafen, Germany). 4-Dimethylaminopyridine (DMAP), N, N'-dicyclohexylcarbodimide (DCC) were purchased from GL Biochem Ltd. (Shanghai, China). Cystamine (CA) dihydrochloride (purity $\geq 96 \%$ ) and Coumarin 6 were purchased from Sigma-Aldrich (St Louis, MO, USA). Pyrene was obtained from Baoman Bio (Shanghai, China).

\section{Chemical compounds}

Bru (PubChem CID: 73,432); LA (PubChem CID: $52,80,450)$. Bru with purity $>98 \%$ was provided by Prof Zhixiu Lin at the School of Chinese Medicine, The Chinese University of Hong Kong. LA was purchased from Aladdin Industrial Corporation (Shanghai, China).

\section{Synthesis of F68-SS-LA copolymer}

The synthesis of the F68-SS-LA conjugate consisted of 3 steps (Figure 1). 1) F68 (1 mmol) and succinic anhydride (SA, $2.2 \mathrm{mmol}$ ) were dissolved in dimethyl sulfoxide (DMSO; Sigma-Aldrich) and reacted under nitrogen protection in the presence of DCC $(2.5 \mathrm{mmol})$ and DMAP $(0.2 \mathrm{mmol})$. After $24 \mathrm{~h}$, the reaction mixture was dialyzed in a dialysis tube with molecular weight cut-off (MWCO) 5000, and the F68-(SA) (COOH-SA-PEO-PPO-PEO-SA$\mathrm{COOH})$ crude products were obtained by lyophilization. 2) CA dihydrochloride was transformed into the CA base form by stirring with $1 \%$ triethylamine for $3 \mathrm{~h}$. CA was conjugated with F68-SA by amidation in the presence of 1-ethyl-3-(3-dimethylaminopropyl)-carbodiimide (EDC) and N-hydroxysuccinimide (NHS) (molar ratio was 2:1:2.5:2.5). The excess CA, DCC, and DMAP were removed by dialyzing in a dialysis tube with MWCO 3500. F68-(CA) $)_{2}$ containing disulfide bonds was obtained by separation using thin-layer chromatography with a mobile phase of methanol/chloroform (70:30, v/v). 3) Finally, 2-fold equivalent molar of LA was conjugated with F68-(CA) 2 to produce EDC/NHS, mediated by amidation conjugation between activated carboxyl group of LA and the terminal amino group of F68-(CA) . After stirring for $24 \mathrm{~h}$, the insoluble by-products were removed using a membrane filter $(0.45 \mu \mathrm{m})$. 


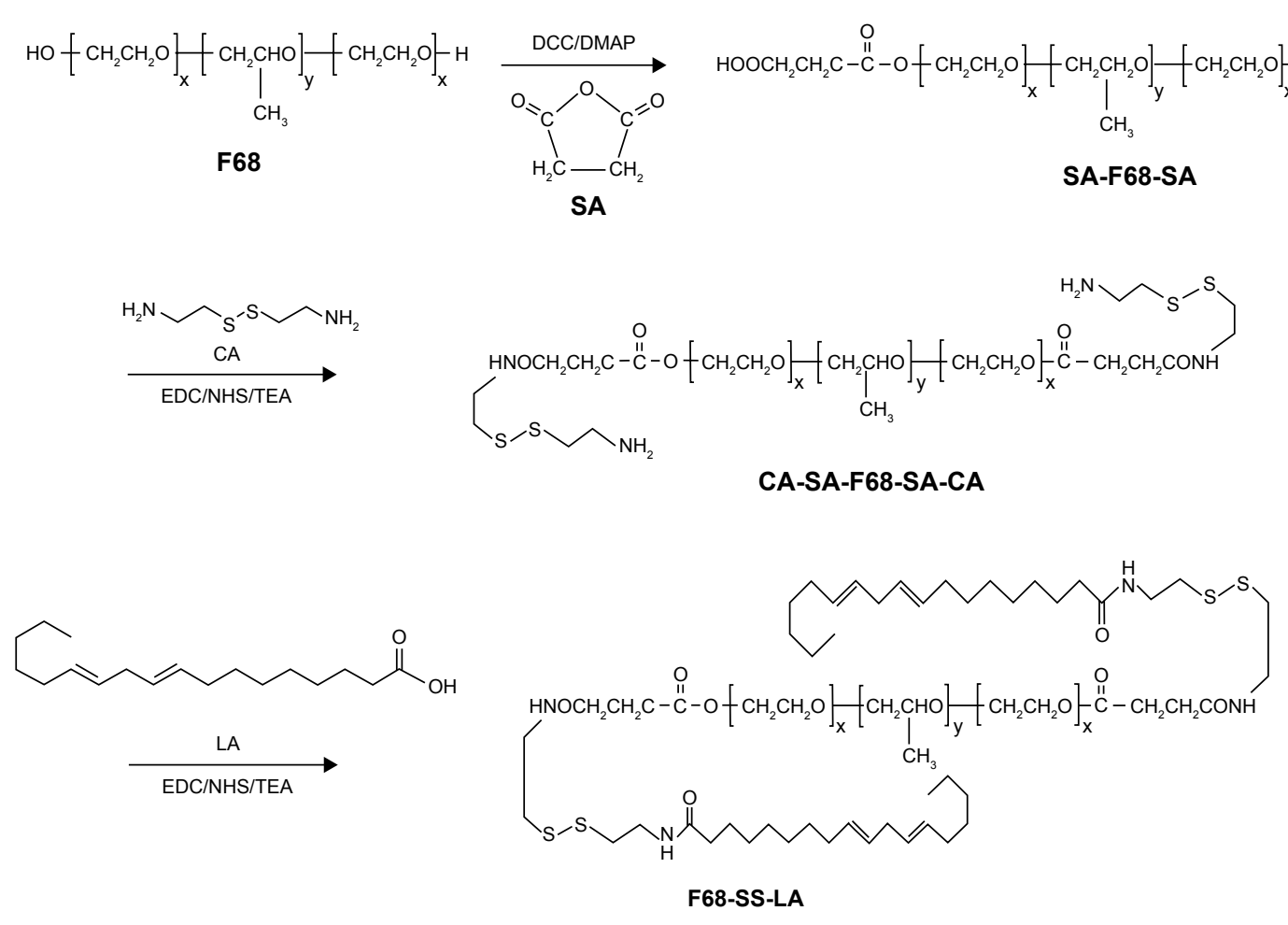

Figure I Synthetic scheme of F68-SS-LA copolymer.

Abbreviations: CA, cystamine; DCC, N, N'-dicyclohexylcarbodimide; DMAP, 4-dimethylaminopyridine; EDC, I-(3-Dimethylaminopropyl)-3-ethylcarbodiimide hydrochloride; LA, linoleic acid; NHS, N-hydroxysuccinimide; SA, succinic acid; TEA, triethylamine.

To compare the redox-sensitivity, F68-CC-LA was also synthesized, as shown in Figure S1. The synthesized copolymers were characterized by ${ }^{1} \mathrm{H}-\mathrm{NMR}$ spectroscopy (400 MHz; Bruker, Bremen, Germany) with deuterated chloroform as the solvent.

\section{Drug loading in F68-SS-LA micelles}

Bru was loaded in F68-SS-LA micelles by film hydration. ${ }^{31}$ Concisely, F68-SS-LA (15 mg) and $2 \mathrm{mg}$ Bru were mixed and dissolved in $2 \mathrm{~mL}$ of methanol. Afterwards, the methanol solution was evaporated using a rotary vacuum to form a thin film, followed by vacuum drying at $40^{\circ} \mathrm{C}$ overnight. The thin film was rehydrated in $10 \mathrm{~mL}$ of deionized water by stirring for $1 \mathrm{~h}$ to form a micellar suspension. The unencapsulated Bru was removed using a membrane filter $(0.45 \mu \mathrm{m})$. Bruloaded F68-SS-LA micelles were further sterilized using a $0.22 \mu \mathrm{m}$ syringe. Blank micelles were prepared using a similar procedure without adding Bru drug. Furthermore, Bru/F68-LA micelles were also prepared by using F68-LA copolymer instead of F68-SS-LA copolymer.

The drug concentration of the Bru-loaded micelles was determined by high-performance liquid chromatography (HPLC) with a maximum absorption wavelength of $360 \mathrm{~nm}$. Micelles were dissolved in an equivalent amount of methanol to disrupt the polymeric shells and to release drugs. The DL amount was determined using a Waters e2695 HPLC with a reversed-phase $\mathrm{C}_{18}$ column $(250 \times 4.6 \mathrm{~mm})$ at a flow rate of $1 \mathrm{~mL} / \mathrm{min}$. The mobile phases consisted of methanol: acetonitrile: $0.25 \%$ acetic acid at 65:30:5. The DL and EE were calculated using the following equations:

$$
\begin{gathered}
\mathrm{DL} \%=\frac{\text { Amount of drug in micelles }}{\text { Amount of feeling polymer and drug }} \times 100 \\
\mathrm{EE} \%=\frac{\text { Amount of drug in micelles }}{\text { Amount of feeling drug }} \times 100
\end{gathered}
$$

\section{Characterization of Bru-loaded redox- sensitive micelles}

The average particle size, size distribution, and zeta potential of the Bru-loaded F68-SS-LA micelles $(1 \mathrm{mg} / \mathrm{mL})$ were measured using a dynamic light scattering (DLS) instrument (Zetasizer Nano ZSP system; Malvern Zetasizer, Malvern, UK) at $25^{\circ} \mathrm{C}$. The morphologies of Bru-loaded micelles containing disulfide linkages were imaged by transmission electron microscopy (TEM) (HRTEM, Tecnai G20; FEI Company, Hillsboro, OR, USA). Specimens were prepared by adding $50 \mu \mathrm{L}$ of micellar suspension dropwise to a Formvar/carbon film grid followed by partial air-drying and negative staining with $2 \%$ phosphotungstic acid. 
The CMC values of the F68-SS-LA and F68 copolymers were determined by fluorescence measurements using pyrene as a hydrophobic probe. ${ }^{32}$ Pyrene solutions $\left(6 \times 10^{-6} \mathrm{M}\right)$ in acetone were added to each vial and were subsequently evaporated to remove the organic solvent. A series of concentrations of copolymer solutions $10 \mathrm{~mL}$ ranging from 0.0001 to $1 \mathrm{mg} / \mathrm{mL}$ was added to the vials and mixed for $3 \mathrm{~h}$ by sonicating and vortexing to equilibrate the pyrene and the micelles. The final concentration of pyrene was $6.0 \times 10^{-7} \mathrm{M}$. The emission spectra of pyrene were recorded from 360 to $450 \mathrm{~nm}$ using a fluorescence spectrophotometer (LUMINA; Thermo Scientific, Waltham, MA, USA) at an excitation wavelength of $339 \mathrm{~nm}$, in which the slit opening for the excitation and emission spectra was set at $5 \mathrm{~nm}$. The intensity ratio of the fluorescence intensities at 373 and $384 \mathrm{~nm}$ in the pyrene emission spectra was then calculated.

The storage stability of Bru-loaded F68-SS-LA micelles (Bru/SS-M) was examined by monitoring the particle size change over the storage time in PBS buffer with or without $10 \%$ fetal bovine serum (FBS). Briefly, Bru/SS-M was lyophilized and redissolved in buffer at $0.5 \mathrm{mg} / \mathrm{mL}$. The particle size and zeta potential of micelle suspensions stored at $37^{\circ} \mathrm{C}$ and $4{ }^{\circ} \mathrm{C}$ were determined by DLS for 7 days.

\section{Redox-sensitivity of Bru-loaded micelles}

The in vitro redox-sensitivity of Bru/SS-M was studied via reduction-triggered disintegration and drug release. Briefly, Bru/SS-M was dissolved in PBS containing $10 \mathrm{mM}$ dithiothreitol (DTT) at $1 \mathrm{mg} / \mathrm{mL}$ and kept in a shaking bath at $37^{\circ} \mathrm{C}$. The particle size was measured by DLS at a predetermined time to monitor disintegration, and a TEM image of Bru/ SS-M with $10 \mathrm{mM}$ DTT treatment for $48 \mathrm{~h}$ was obtained.

In vitro release of Bru from F68-SS-LA micelles was studied using a dialysis method, in which $0.5 \%$ Tween- 80 solution was used as the release medium. Briefly, $2 \mathrm{~mL}$ Bru-loaded micelle suspension was added to a dialysis bag with MWCO $3.5 \mathrm{kDa}$ and was incubated in $40 \mathrm{~mL}$ of release medium with or without $10 \mathrm{mM}$ DTT with shaking at $100 \mathrm{rpm}$ and $37^{\circ} \mathrm{C}$. The drug-leakage profile of an equivalent amount of free Bru was employed as control. At predetermined time points, $2 \mathrm{~mL}$ of release medium was collected, and the same volume of fresh release medium was added. The concentration of Bru in the release medium was determined by HPLC. The cumulative percentage of Bru release was calculated using the following formula:

$$
\mathrm{M}_{\mathrm{i}}=\mathrm{C}_{\mathrm{i}} \times 40 \mathrm{~mL}+\sum_{\mathrm{j}=1}^{\mathrm{j}=\mathrm{j}-1} \mathrm{C}_{\mathrm{j}} \times 1 \mathrm{~mL}
$$

$$
\text { Cumulative release }(\%)=\left(\mathrm{M}_{\mathrm{i}} / \mathrm{M}_{\mathrm{A}}\right) \times 100
$$

$\mathrm{M}_{\mathrm{i}}$ represents the total amount of drug released at the ith time point. $\mathrm{C}_{\mathrm{i}}(\mathrm{mg} / \mathrm{mL})$ represents the concentration of Bru in the release medium at the ith time point. $\sum_{\mathrm{j}=1}^{\mathrm{j}=\mathrm{j}-1} \mathrm{C}_{\mathrm{j}} \times 1 \mathrm{~mL}$ represents the total amount of drug release before the jth time point. $M_{A}$ represents the initial amount of Bru in the dialysis bag.

\section{Cell culture and drug treatment}

Human hepatocellular carcinoma Bel-7402 and breast cancer MCF-7 cell lines, obtained from American Type Culture Collection, were cultured in DMEM with high glucose containing $10 \% \mathrm{FBS}$, supplemented with $50 \mathrm{U} \cdot \mathrm{mL}^{-1}$ penicillin-streptomycin (Invitrogen, Carlsbad, CA, USA) and incubated at $37^{\circ} \mathrm{C}$ in a $5 \% \mathrm{CO}_{2}$ atmosphere. Cells were passaged with $0.05 \%$ trypsin-EDTA (Invitrogen). Cells pretreated with samples were seeded into 12-well plates or 96-well plates 1 day before the experiment. Bru was dissolved in sterile DMSO and stored at $4^{\circ} \mathrm{C}$. The micellar suspension was filtered with a $0.22 \mu \mathrm{M}$ pore PVDF syringe filter for sterilization. Solvent control was included by diluting the same volume of DMSO or PBS buffer used in drug preparation in the culture medium.

\section{Cell uptake studies}

The cellular uptake efficiency of Bru micelles in Bel-7402 and MCF-7 cells was measured by determining the intracellular drug concentration via HPLC. Briefly, cells were seeded in a 6-well plate at a density of $1 \times 10^{5}$ cells per well for $24 \mathrm{~h}$ before the experiment. Cells were treated with free Bru, Bru/F68-CC-LA (non-SS-M), and Bru/F68-SS-LA (SS-M) (equivalent Bru concentration of $2 \mu \mathrm{M}$ ) for predetermined time intervals $\left(1,3\right.$, and $6 \mathrm{~h}$, respectively) at $37^{\circ} \mathrm{C}$. At the end of the incubation period, cells were collected and washed 3 times with cold PBS. Then, cells were lysed with $1 \%$ Triton $\mathrm{X}-100$. Both the protein amount and Bru concentration in the cell lysate were determined using a BCA protein assay kit and HPLC analysis, as previously reported. ${ }^{33}$ Cellular accumulation of Bru was normalized with respect to the total protein amount.

In addition, the fluorescent dye coumarin-6 (C6) was employed to replace Bru and was loaded in micelles to visualize the cellular internalization capacity. The cellular uptake of C6/NPs in Bel-7402 and MCF-7 cells was observed using an IN Cell Analyzer (GE Healthcare, Little Chalfont, UK). After incubation with cells for $6 \mathrm{~h}$, culture medium containing the 
aforementioned $\mathrm{C} 6$ samples with an equivalent concentration of C6 $\left(1 \mu \mathrm{g} \cdot \mathrm{mL}^{-1}\right)$ was removed. Cells were washed 3 times with cold PBS, fixed in 4\% paraformaldehyde for $10 \mathrm{~min}$, stained with Hoechst 33342 for $10 \mathrm{~min}$, and observed by fluorescent DAPI and fluorescein isothiocyanate (FITC) channels, respectively.

\section{Cell viability}

The in vitro cytotoxicity of Bru/SS-M was investigated on Bel-7402 and MCF-7 cells using the 3-(4 5-dimethylthiazol2-yl)-2 5-diphenyltetrazolium bromide (MTT) assay. Briefly, $5 \times 10^{3}$ cells were seeded in each well of a 96 -well plate. After $24 \mathrm{~h}$ incubation, cells were treated with free Bru, Bru/ non-SS-M, and Bru/SS-M at indicated concentrations for 24 and $48 \mathrm{~h}$. The untreated cells acted as controls. After treatment, cells were kept in fresh culture medium containing $1 \mathrm{mg} / \mathrm{mL}$ of MTT for another $4 \mathrm{~h}$ at $37^{\circ} \mathrm{C}$. DMSO $100 \mu \mathrm{L}$ was added to dissolve the formed formazan crystals. The optical density was recorded at $570 \mathrm{~nm}$ on a microplate reader. Each treatment group included 3 individual wells, and cell viability was calculated as $\mathrm{OD}_{\text {treatment }} / \mathrm{OD}_{\text {control }}$.

\section{Mitochondrial membrane potential (MMP) assay}

The change in MMP was determined by the $5,5^{\prime}, 6,6^{\prime}$ tetrachloro-1,1',3,3'-tetraethylbenzimidazolcarbocyanine iodide (JC-1) probe and analyzed using flow cytometry (FCM). Bel-7402 and MCF-7 cells were plated in 12-well plates at a density of $5 \times 10^{4}$ cells per well. After treatment with various Bru formulations (equivalent concentration of $5 \mu \mathrm{M}$ ) for $12 \mathrm{~h}, 5 \mu \mathrm{L} \cdot \mathrm{mL}^{-1}$ of the $\mathrm{JC}-1$ staining solution (Beyotime, Shanghai, China) was added to each well followed by co-incubation for $20 \mathrm{~min}$ in the dark. The untreated cells and cells treated with $0.1 \%$ carbonyl cyanide $\mathrm{m}$-chlorophenylhydrazone acted as the negative and positive controls, respectively. Cells were washed, collected, and resuspended in $200 \mu \mathrm{L}$ JC-1 working buffer. The ratio of red fluorescence to green fluorescence was defined as the depolarization level.

\section{Reactive oxygen species (ROS) generation}

The accumulation of intracellular ROS was detected using the $2^{\prime}, 7^{\prime}$-dichlorofluorescin diacetate $\left(\mathrm{DCFH}_{2}-\mathrm{DA}\right)$ probe, as described previously. ${ }^{34}$ Briefly, after treatment with various Bru formulations (equivalent concentration of $5 \mu \mathrm{M}$ ) for $6 \mathrm{~h}$, cells were co-incubated with $1 \mu \mathrm{M} \mathrm{DCFH}_{2}$-DA for $20 \mathrm{~min}$ at $37^{\circ} \mathrm{C}$ in a humidified atmosphere at $5 \% \mathrm{CO}_{2}$. Cells were washed, collected, and resuspended in $200 \mu \mathrm{L}$ PBS in the dark. To quantify ROS, the fluorescence intensity (FL-1 channel) was measured by FCM. The green fluorescence generated by the accumulated ROS was observed using a fluorescence microscope.

\section{Apoptosis assay}

The apoptosis induction effect of Bru/F68-SS-LA in Bel7402 and MCF-7 cells was detected by Annexin V-FITC/ propidium iodide (PI) assay. Briefly, cells were plated into 6 -well plates with $2 \times 10^{5}$ cells per well and treated for $24 \mathrm{~h}$ with Bru formulations (equivalent concentration of $5 \mu \mathrm{M}$ ). Subsequently, cells were collected, washed with cold PBS, successively stained with $3 \mu \mathrm{L}$ of Annexin V-FITC and 2 $\mu \mathrm{L}$ of $100 \mu \mathrm{g} / \mathrm{mL}$ PI, and resuspended in $200 \mu \mathrm{L}$ of binding buffer. After 15 min incubation, a total of at least 10,000 events were recorded by FCM (BD, San Diego, CA, USA).

Calcein-AM/PI double staining was used to evaluate cell death. ${ }^{35}$ Similarly, Bel-7402 and MCF-7 cells were seeded in 96-well plates and treated with samples with an equivalent concentration of Bru $(2 \mu \mathrm{M})$. After $24 \mathrm{~h}$, the cells were washed and stained with calcein-AM/PI for $10 \mathrm{~min}$. Cells were observed using an IN Cell Analyzer (GE Healthcare) in the green and red fluorescent channels, which provided quantification of living and dead cells, respectively.

\section{Statistical analysis}

Data are presented as the mean \pm SD. Statistical significance was determined by analysis of variance among 3 groups or Student's $t$-test between 2 groups. $P$-values of $<0.05$ were considered significant.

\section{Results and discussion Characterization of the F68-SS-LA conjugate}

The F68-SS-LA conjugate was synthesized by multiple graft reactions, as shown in Figure $1 .{ }^{1} \mathrm{H}$ NMR spectra were used to confirm the process (Figure 2A). We observed typical signals for the F68 polymer, including the $-\mathrm{CH}_{2}$ peaks $(\delta=3.60-3.65 \mathrm{ppm}),-\mathrm{CH}$ peaks $(\delta=3.50-3.55 \mathrm{ppm})$, and the $-\mathrm{CH}_{3}$ peaks $(\delta=1.10-1.17 \mathrm{ppm})$. Additionally, we observed the typical signals for LA in the spectra of F68SS-LA, including peaks a $(\delta=5.32-5.39$ ppm $)$, peaks $b$ $(\delta=2.75-2.80 \mathrm{ppm})$, peaks c $(\delta=2.30-2.36 \mathrm{ppm})$, peaks $d(\delta=2.03-2.13 \mathrm{ppm})$, peaks e $(\delta=1.59-1.66 \mathrm{ppm})$, peaks $\mathrm{f}(\delta=1.28-1.39 \mathrm{ppm})$, and peaks $\mathrm{g}(\delta=0.87-0.93 \mathrm{ppm})$. 

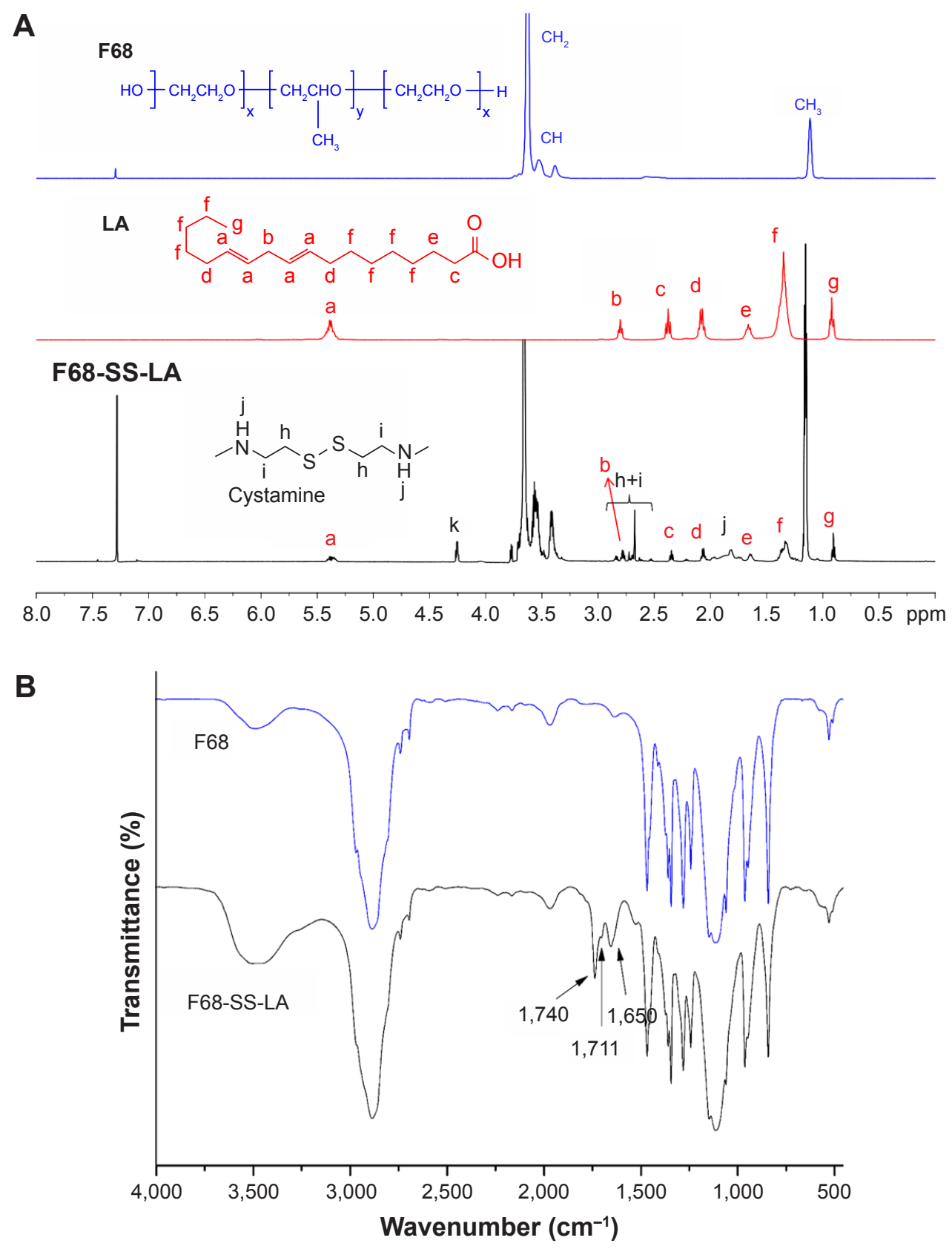

Figure 2 (A) 'H NMR spectra of F68 polymer, LA moiety, and F68-SS-LA copolymer; (B) FT-IR spectra of F68 polymer and F68-SS-LA copolymer. Abbreviations: LA, linoleic acid; FT-IR, Fourier transform-infrared spectroscopy; NMR, nuclear magnetic resonance.

Because of the successful conjugation, all the representative peaks were observed in the ${ }^{1} \mathrm{H}$ NMR spectrum of F68-SS-LA. Moreover, the methylene peaks of $\mathrm{CA}\left(-\mathrm{C}_{2}-\mathrm{CH}_{2}-\mathrm{S}-\mathrm{S}-\right.$ $\mathrm{C}_{2}-\underline{\mathrm{CH}}_{2}-$ ) at $\delta 2.71-2.86 \mathrm{ppm}$ (peak h and i) ${ }^{36}$ that emerged in F68-SS-LA demonstrated the successful synthesis of the F68-SS-LA conjugate. In addition, the Fourier transforminfrared (FT-IR) spectra of F68-SS-LA further supported the verification of the F68-SS-LA polymer structure (Figure 2B). Based on the FT-IR spectrum of LA, ${ }^{37}$ the stretching bands at $1,740,1,711$, and $1,650 \mathrm{~cm}^{-1}$ resulted from amides (-CO-NH-) in the F68-SS-LA conjugates. The ${ }^{1} \mathrm{H}$ NMR spectrum of the F68-SS-LA copolymer is shown in Figure S2.

\section{Preparation and characterization of Bru-loaded micelles}

The amphiphilic nature of the F68-SS-LA copolymer enabled micelles to be generated by self-assembly. The blank F68SS-LA micelles exhibited a particle size of $83.56 \pm 3.42 \mathrm{~nm}$ and a zeta potential at $-16.8 \pm 1.2 \mathrm{mV}$. According to our previous study, the apparent partition coefficient $(\log \mathrm{P})$ of 
Bru was $\sim 1.82$. Thus, hydrophobic Bru could be encapsulated into F68-SS-LA micelles through hydrophobic interactions between the LA moieties and Bru agents, with the narrow size distribution for the EPR effect (Figure 3A). With a Bru feed ratio of $11.7 \%(\mathrm{w} / \mathrm{w})$, the DL and EE in the F68SS-LA micelles were $8.4 \% \pm 0.3 \%(\mathrm{w} / \mathrm{w})$ and $92.0 \% \pm 0.5 \%$, respectively. Based on DLS determination, Bru/F68-SS-LA micelles possessed a narrow average size distribution of $94.38 \pm 2.68 \mathrm{~nm}$ (polydispersity index $[\mathrm{PDI}]<0.2$ ) with a zeta potential of $-17.2 \pm 1.6 \mathrm{mV}$. Similarly, blank F68-SS-LA micelles exhibited a narrow average size distribution of $88.42 \pm 2.12 \mathrm{~nm}$ with a PDI value of 0.146 . The TEM images in Figure 3B show that the drug-loaded micelles were spherical in shape with sizes of $50-100 \mathrm{~nm}$. The size range of the micelles indicated that Bru-M was selectively accumulated in solid tumors via the EPR effect. ${ }^{38}$

The self-assembly behavior of the F68-SS-LA copolymer in aqueous solution and the dilution resistance capacity were characterized based on the CMC and compared with the F68-CC-LA block polymer. The fluorescent spectrum
A
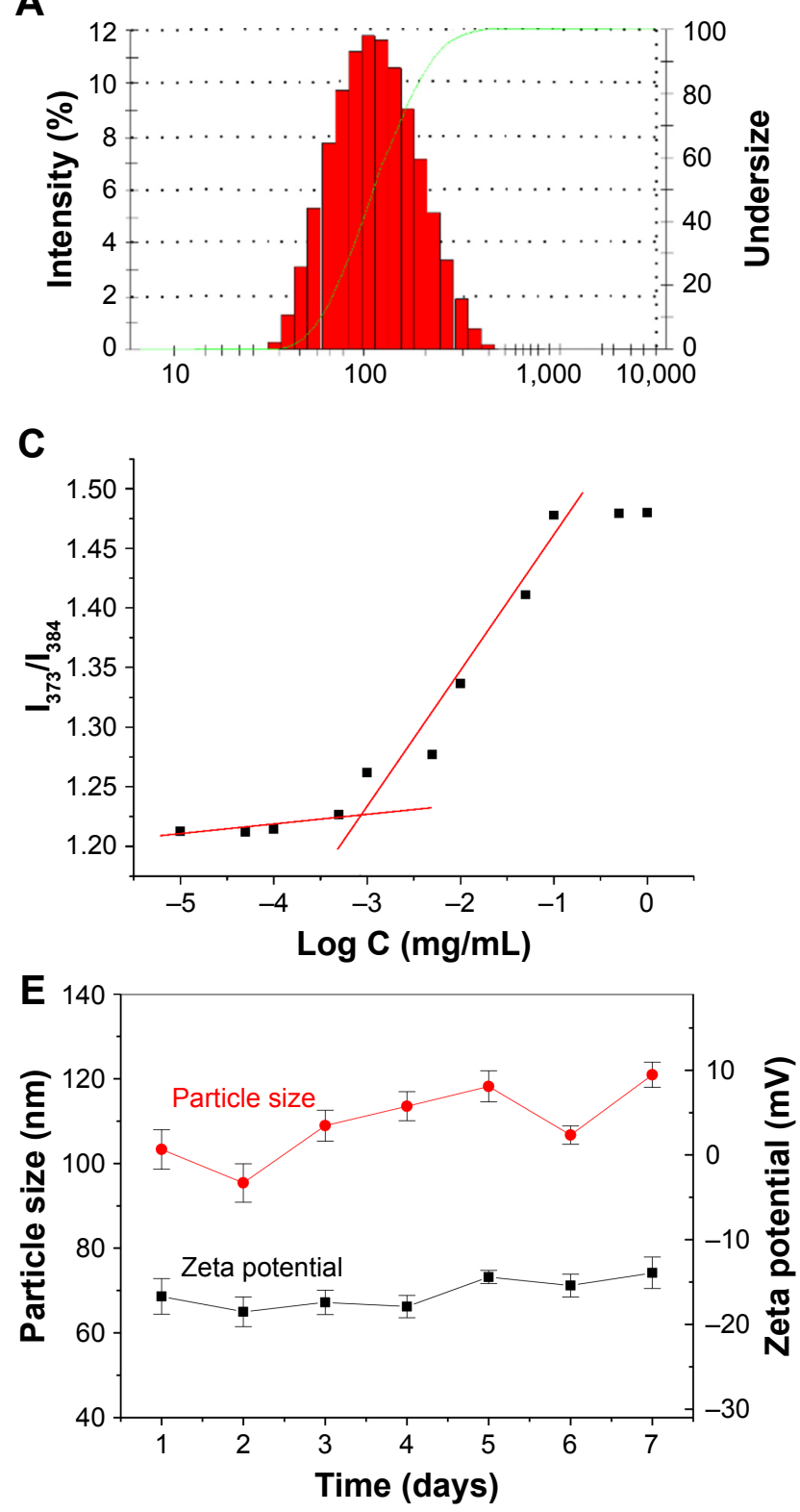

B
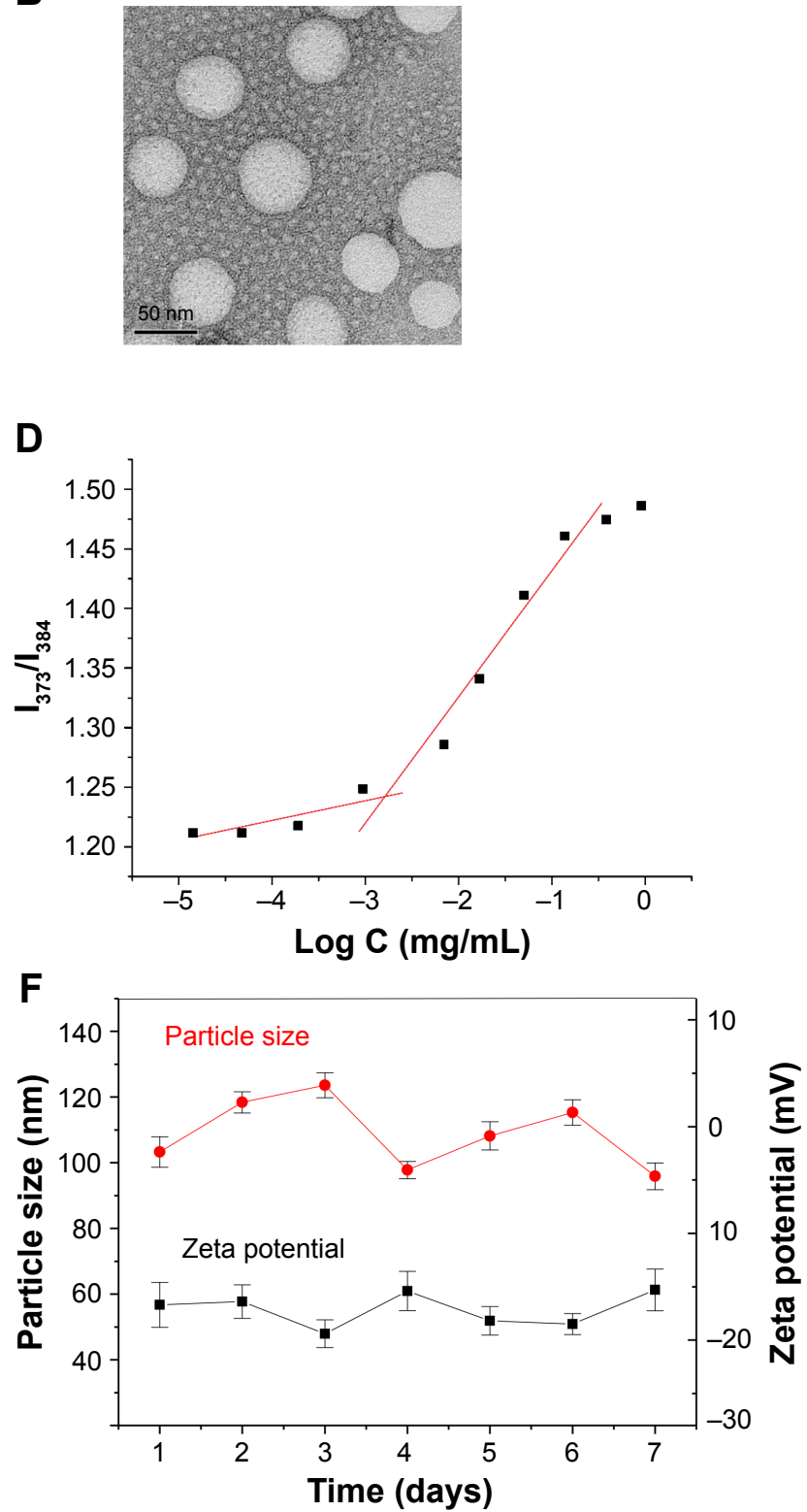

Figure 3 Characterization of Bru-loaded F68-SS-LA micelles (Bru/SS-M).

Notes: (A) Particle size distribution by DLS determination. (B) TEM images of Bru/SS-M, scale bar is 50 nm. Magnification $\times 250,000$. Intensity ratio of $I_{373} / I_{384}$ from emission spectra of pyrene as a function of log (F68-SS-LA). (C) and F68-CC-LA (D) polymer concentration for CMC value determination. Storage stability evaluation of Bru/SS-M in particle size and zeta potential at $37^{\circ} \mathrm{C}(\mathbf{E})$ and $4^{\circ} \mathrm{C}(\mathbf{F})$.

Abbreviations: Bru, brusatol; DLS, dynamic light scattering; LA, linoleic acid; CMC, critical micelle concentration; TEM, transmission electron microscopy. 
of pyrene probe will change if the pyrene loaded in the micelle core is released into the aqueous solution when the micelles are diluted excessively. The CMC was obtained by plotting the ratio of $\mathrm{I}_{373} / \mathrm{I}_{384}$ of the emission spectra profile vs the concentration of copolymers. As shown in Figure 3C and $\mathrm{D}$, this ratio decreased with increasing concentration of copolymer. The CMCs for F68-SS-LA and F68-CC-LA were as low as $7.94 \times 10^{-4}$ and $15.48 \times 10^{-4} \mathrm{~g} / \mathrm{L}$, respectively. This similar CMC values of the F68-SS-LA and F68-CC-LA copolymers indicated that there was no significant difference in the self-assembly behavior of F68 conjugates with redoxsensitive linkage and nonredox-sensitive linkage. However, the copolymers conjugated with LA moieties exhibited a much lower CMC than a single F68 polymer (CMC for F68 was $\sim 1.003 \times 10^{-2} \mathrm{~g} / \mathrm{L}$ in the previous study $\left.{ }^{39}\right)$. The addition of LA moiety to the $\mathrm{F} 68$ polymer could significantly decrease the CMC of F68, resulting in higher stability and antidilution capacity.

Subsequently, the storage stability of Bru/F68-SS-LA micelles at $37^{\circ} \mathrm{C}$ and $4{ }^{\circ} \mathrm{C}$ was monitored using DLS to determine the change in size and zeta potential. As shown in Figure $3 \mathrm{E}$ and F, the properties of Bru/F68-SS-LA micelles did not change significantly after 1 week of storage, with no precipitation or aggregation observed.

\section{Redox-sensitivity of micelles}

The size change of Bru/SS-M in buffer containing $10 \mathrm{mM}$ DTT at different time intervals was detected to determine the reduction-triggered destabilization. During a $48 \mathrm{~h}$ incubation,
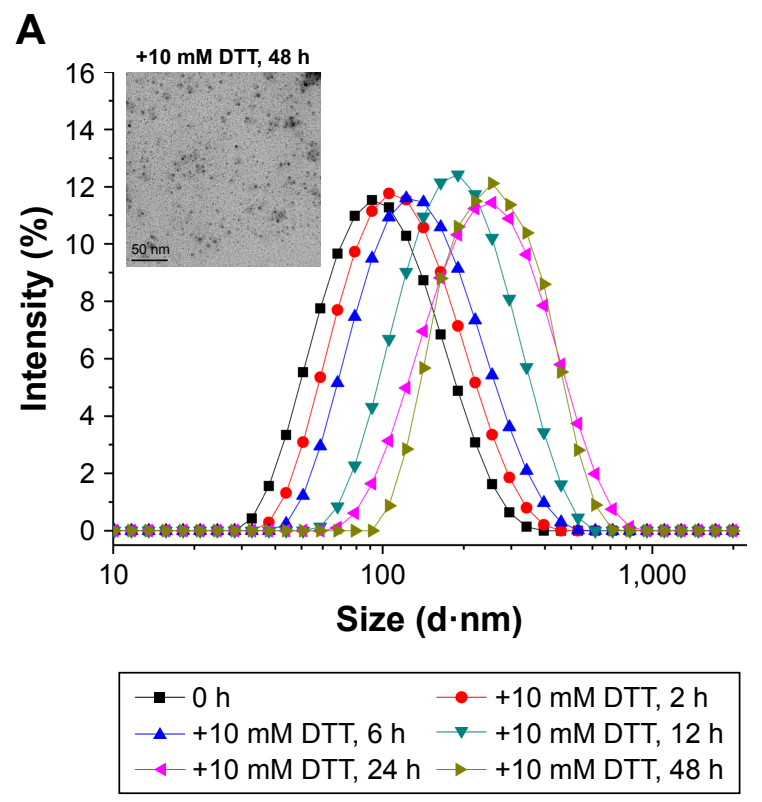

a gradual increase in the size of the micelles was observed (Figure 4A). The initial size of Bru/SS-M showed a unimodal distribution with an average hydrodynamic diameter of $\sim 94 \mathrm{~nm}$. However, the particle size of the micelles dramatically increased to $>450 \mathrm{~nm}$ after $48 \mathrm{~h}$ incubation with $10 \mathrm{mM}$ DTT. After $48 \mathrm{~h}$ of incubation, monodisperse particles were not observed in the TEM image (Figure 4A), indicating the redox-sensitive dissociation of micelles containing SS linkage.

To further investigate the redox-sensitive in vitro release of Bru from F68-SS-LA micelles triggered by destabilization, the Bru release profiles in release medium with or without $10 \mathrm{mM}$ DTT under sink conditions were investigated by dialysis. Bru was released much more rapidly from Bru/SS-M in the presence of $10 \mathrm{mM}$ DTT than without DTT (Figure 4B). After $48 \mathrm{~h}$ of shaking, $80 \%$ of Bru was released from Bru/ SS-M in medium without DTT. However, $\sim 85 \%$ of Bru was released from Bru/SS-M with $10 \mathrm{mM}$ DTT after $12 \mathrm{~h}$, which was similar to the leakage profile of free Bru. Both the fast micellar destabilization and drug release in a high reduction environment suggested that F68-SS-LA micelles could facilitate the rapid increase of the intracellular concentration of Bru in cancer cells.

\section{Cellular uptake in vitro}

The cellular uptake was investigated to determine the capacity of F68-SS-LA micelles to improve the Bru concentration in cancer cells. As shown in Figure 5A and B, a higher concentration of Bru was detected in cells incubated with Bru

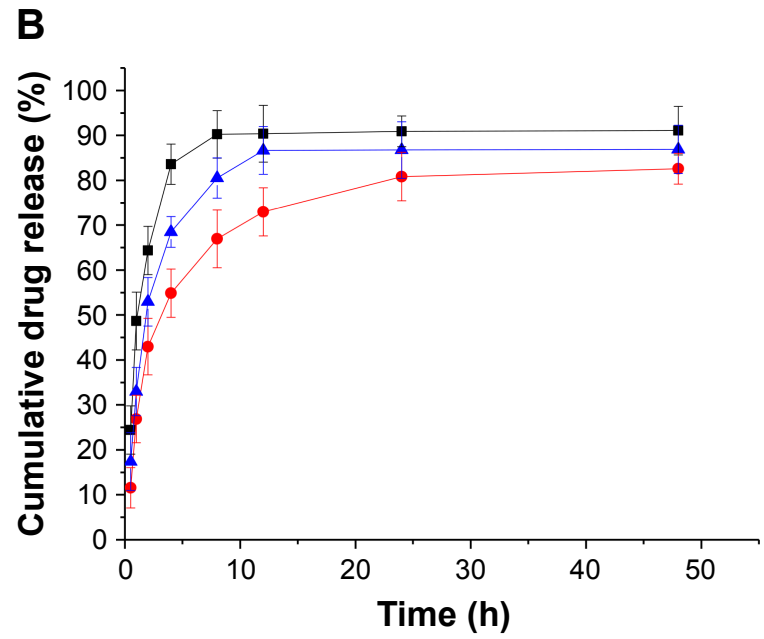

- Free Bru
- Bru/SS-M
$\longleftarrow$ Bru/SS-M + 10 mM DTT

Figure 4 Redox-sensitive size change. Magnification $\times 250,000$. (A) and drug release in vitro (B) of Bru/SS-M in presence of $10 \mathrm{mM}$ DTT. Abbreviations: Bru, brusatol; DTT, dithiothreitol. 
A

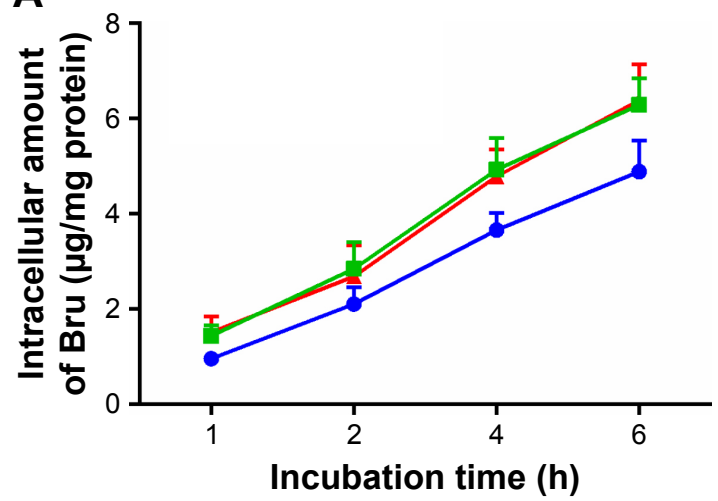

B

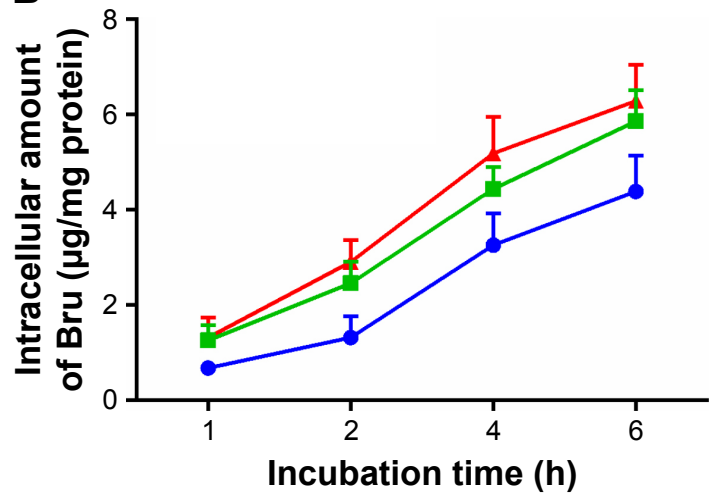

$\rightarrow-$ Free Bru $\rightarrow-$ Bru/non-SS-M $\simeq$ Bru/SS-M

C
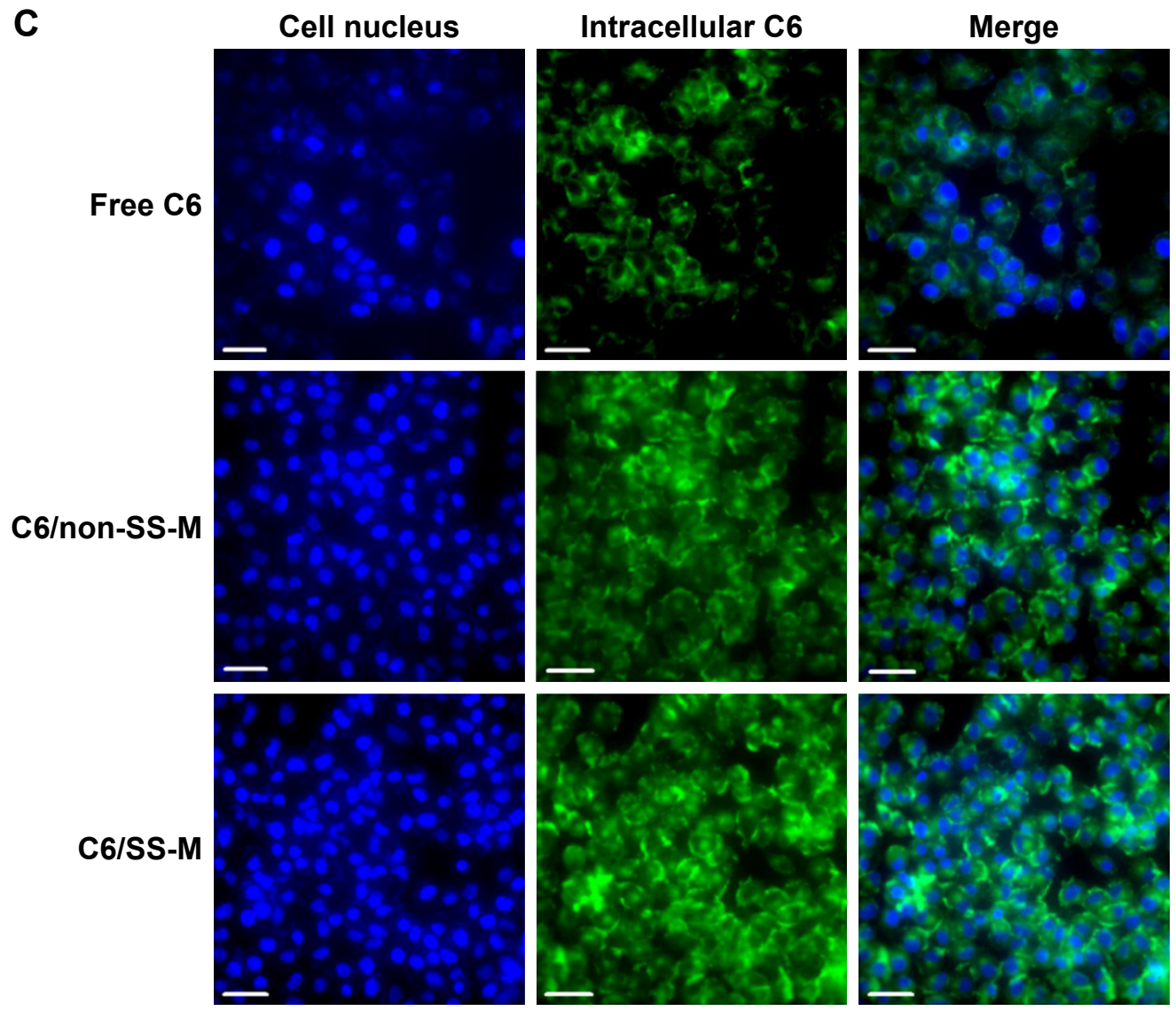

Figure 5 Cellular uptake of Bru/SS-M in Bel-7402 cells (A) and MCF-7 cells (B) after I, 2, 4, 6 h incubation. (C) Fluorescence imaging of C6-loaded micelles in Bel-7402 cells after $6 \mathrm{~h}$ incubation. Cell nucleus was stained by Hoechst 33342 (blue). Scale bar is $50 \mu \mathrm{m}$. Magnification $\times 100$.

Abbreviation: Bru, brusatol.

micelles than that of free Bru at each time point due to the higher penetration capacity of the nanosized carriers. In contrast, no difference in cellular uptake between Bru/SS-M and Bru/non-SS-M was observed, indicating that disulfide bonds in copolymers would not influence the internalization process of micelles. However, the redox-sensitive drug release behavior of Bru/SS-M could be leveraged to rapidly increase the drug concentration in the cytoplasm. Taken together, the micelle structure and redox-sensitive drug release behavior synergistically improve intracellular drug delivery.

Furthermore, to visualize the cellular uptake of F68SS-LA micelles, the green fluorescent dye C6 was used as a model drug to visually analyze endocytosis and the intracellular distribution in Bel-7402 cells. Figure 5C shows that intracellular C6 mainly distributed around the nucleus. After $6 \mathrm{~h}$ of incubation, more green fluorescence was observed 
in cells treated with both $\mathrm{C6} /$ non-SS-M and C6/SS-M than that of free C6. Moreover, compared with fluorescent dots in the C6/non-SS-M group, homogeneous fluorescence was observed in the cytoplasm of cells treated with C6/SS-M. This finding suggested that the reduction-triggered SS-M had a similar cellular uptake capacity to the non-SS-M counterpart but displayed more efficient intracellular drug release activity. ${ }^{40,41}$ These results demonstrated that redox-sensitive micelles could improve the accumulation of Bru intracellularly, which could lead to higher cytotoxicity against cancer cells.

\section{Cytotoxicity in vitro}

The in vitro cytotoxicity of Bru/SS-M was assessed on Bel7402 and MCF-7 cells using the MTT assay in comparison with Bru in DMSO solution (free Bru) and Bru in nonredoxsensitive micelles (Bru/non-SS-M). Specifically, the cytotoxicity of empty micelles of F68-SS-LA and F68-CC-LA with excess copolymer concentrations as Bru/SS-M and Bru/ non-SS-M were evaluated (Figure S3). After $48 \mathrm{~h}$ of incubation, $10-100 \mu \mathrm{g} / \mathrm{mL}$ of F68-SS-LA and F68-CC-LA empty micelles did not exhibit significant cell survival inhibition, with $>85 \%$ cell viability, indicating good biocompatibility of the designed copolymers. However, free Bru and Bru micelles remarkably decreased the cell survival rate in a doseand time-dependent manner (Figure 6). The cytotoxicity of Bru/non-SS-M was comparable with that of free Bru. However, Bru/SS-M drastically reduced cell viability compared with free Bru and Bru/non-SS-M at all Bru concentrations. For example, after $48 \mathrm{~h}$ of treatment, the $\mathrm{IC}_{50}$ values for free Bru, Bru/non-SS-M and Bru/SS-M were 1.301, 1.158 and $0.634 \mu \mathrm{M}$ on Bel-7402 cells and 1.850, 1.694 and $0.992 \mu \mathrm{M}$ on MCF-7 cells, respectively. This was mainly due to the increased cellular uptake and accelerated drug release in the reductive environment of the cytoplasm. ${ }^{42-44}$ Therefore, delivery via F68-SS-LA micelles could potentially improve the in vitro cytotoxicity of Bru.

\section{Mitochondrial membrane potential}

The determination of the mitochondrial permeability transition profile, that is, variation in MMP, provides an early indication of cellular apoptosis. In cells with high MMP (negative), JC-1 can accumulate in the mitochondria, where JC-1 aggregates exhibit red fluorescence, while JC-1 in the cytoplasm, associated with low MMP and found as monomers, shows green fluorescence. Thus, loss of MMP due to
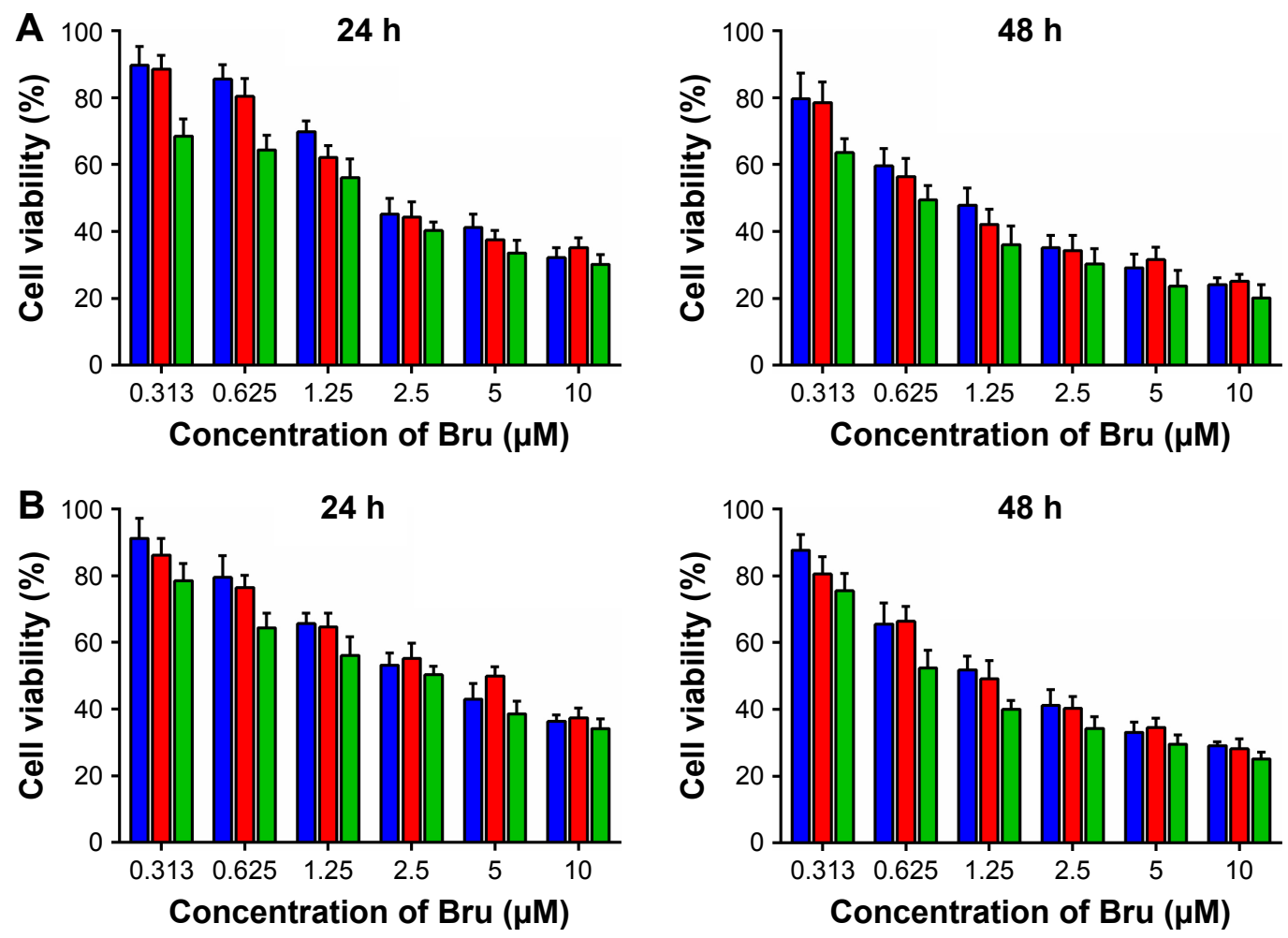

$\square$ Free Bru $\square$ Bru/non-SS-M $\square$ Bru/SS-M

Figure 6 In vitro cytotoxicity of free Bru and Bru-loaded micelles against Bel-7402 cells (A) and MCF-7 cells (B) for 24 and 48 h treatment determined by MTT assay. Abbreviation: Bru, brusatol. 


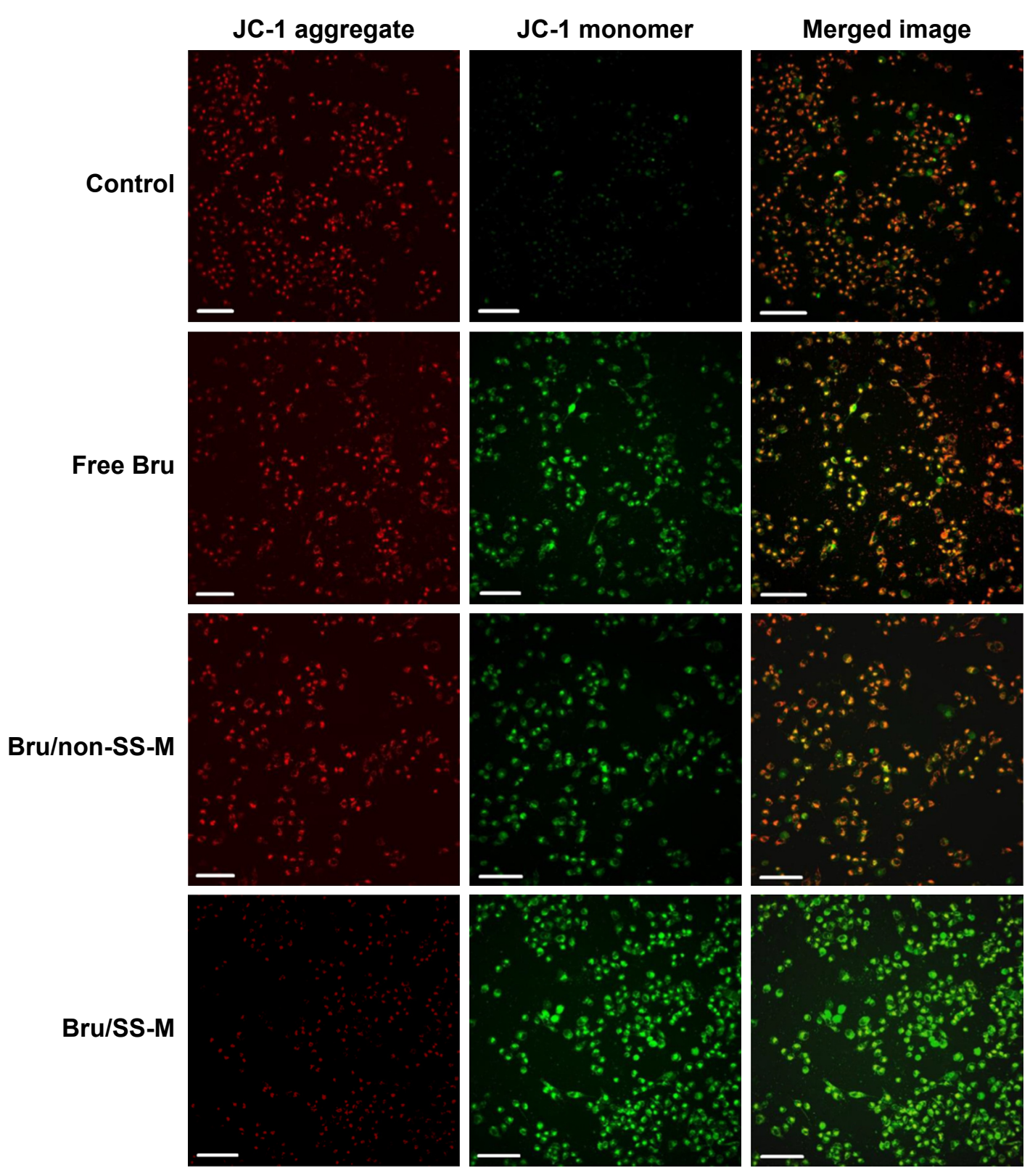

Figure 7 Fluorescence microscope images of Bel-7402 cells treated with various Bru formulations (equivalent Bru concentration of $5 \mu \mathrm{M}$ ) for $12 \mathrm{~h}$ treatment and subsequently stained with JC-I dye. Scale bar is $200 \mu \mathrm{m}$. Magnification $\times 20$.

Abbreviation: Bru, brusatol.

Bru can be detected by the fluorescence variation caused by the change in the JC-1 dye state. As shown in Figure 7, compared with the minimal green fluorescence observed in the negative control group, various Bru formulations resulted in a transition of red fluorescence to green fluorescence in Bel7402 cells, indicating leakage of JC-1 from the mitochondria in association with the decrease in MMP. Particularly, Bru/ SS-M produced much more evident membrane depolarization than the other formulations, which suggested that Bru loaded in F68-SS-LA micelles could remarkably reduce MMP in Bel-7402 and provoke cell death via the initial apoptotic pathway. Similarly, the significant MMP decrease induced by Bru/SS-M was also observed in MCF-7 cells, as shown in Figure S4.

\section{Intracellular ROS generation}

ROS is a well-known mediator of intracellular cascade signals. An excessively increased ROS level could lead to opening of the mitochondrial permeability transition pore and subsequently stimulate pro-apoptosis and DNA damage..$^{45,46}$ To determine whether the cytotoxicity of Bru against cancer cells was associated with ROS, the ROS level was measured 
using the ROS-detecting fluorescent dye $\mathrm{DCFH}_{2}$-DA, where the fluorescent DCF generated by intracellular ROS was detected. The fluorescent intensity of Bel-7402 (Figure 8A) and MCF-7 (Figure 8B) cells treated with Bru formulations was significantly increased. This finding provided evidence that the proliferation inhibition activity of Bru could involve increased ROS generation. Furthermore, treatment with Bru/ SS-M caused significantly higher ROS production than both free Bru and Bru/non-SS-M. Specifically, the ROS level in Bel-7402 cells after treatment with Bru/SS-M was 1.84- and 1.63-fold the levels in the free Bru and Bru/non-SS-M groups, respectively. Similarly, Bru/SS-M resulted in 1.89- and 1.53fold higher ROS production of MCF-7 cells than in the free Bru and Bru/non-SS-M groups, respectively. This indicated that the delivery of Bru by redox-sensitive micelles induced oxidative stress, which played a key role in the cytotoxicity against cancer cells.

\section{Induction of the apoptotic rate}

Apoptosis, one of the major types of cell death, has been identified as the general pathway to halt tumor cell proliferation for most of chemotherapeutic agents. Decreased MMP

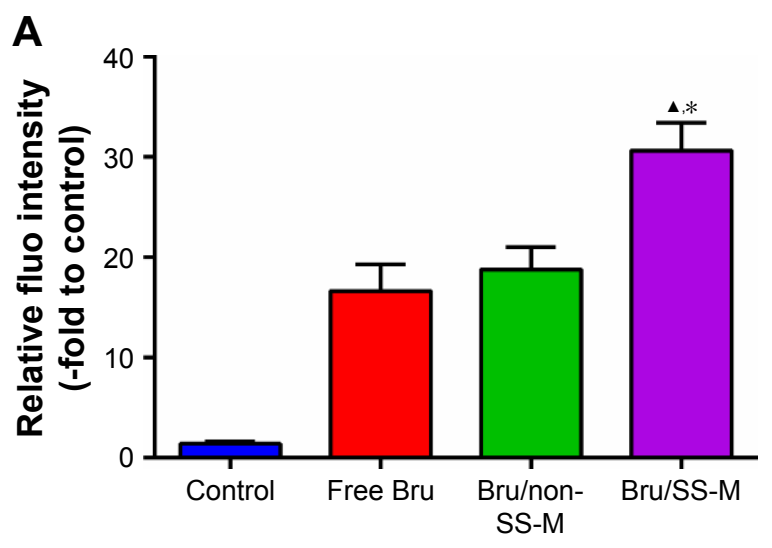

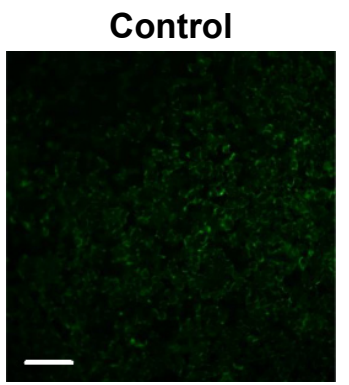
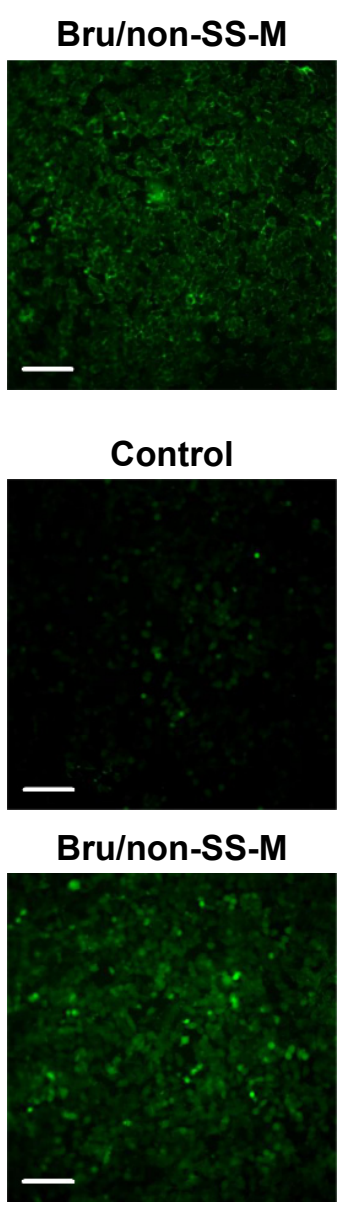

\section{Free Bru}

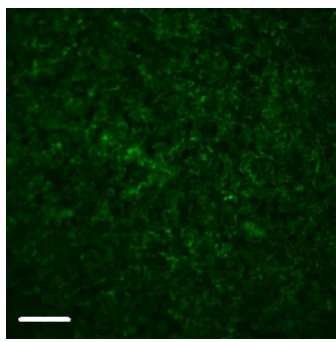

Bru/SS-M

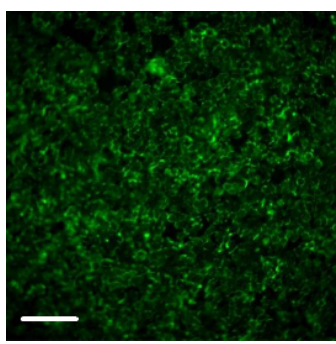

Free Bru

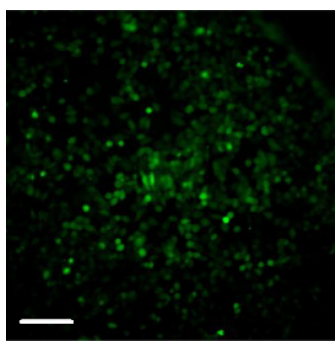

Bru/SS-M

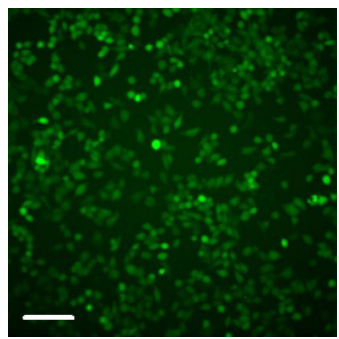

Figure 8 Effect of Bru formulations on ROS level in Bel-7402 (A) and MCF-7 cells (B), by means of quantitative analysis by FCM and observation of the generated fluorescence intensity.

Notes: ${ }^{\star} p<0.05$ Bru/SS-M vs free Bru. ${ }^{*} p<0.05$ Bru/SS-M vs Bru/non-SS-M. Scale bar is $200 \mu \mathrm{m}$.

Abbreviations: Bru, brusatol; FCM, flow cytometry; ROS, reactive oxygen species. 

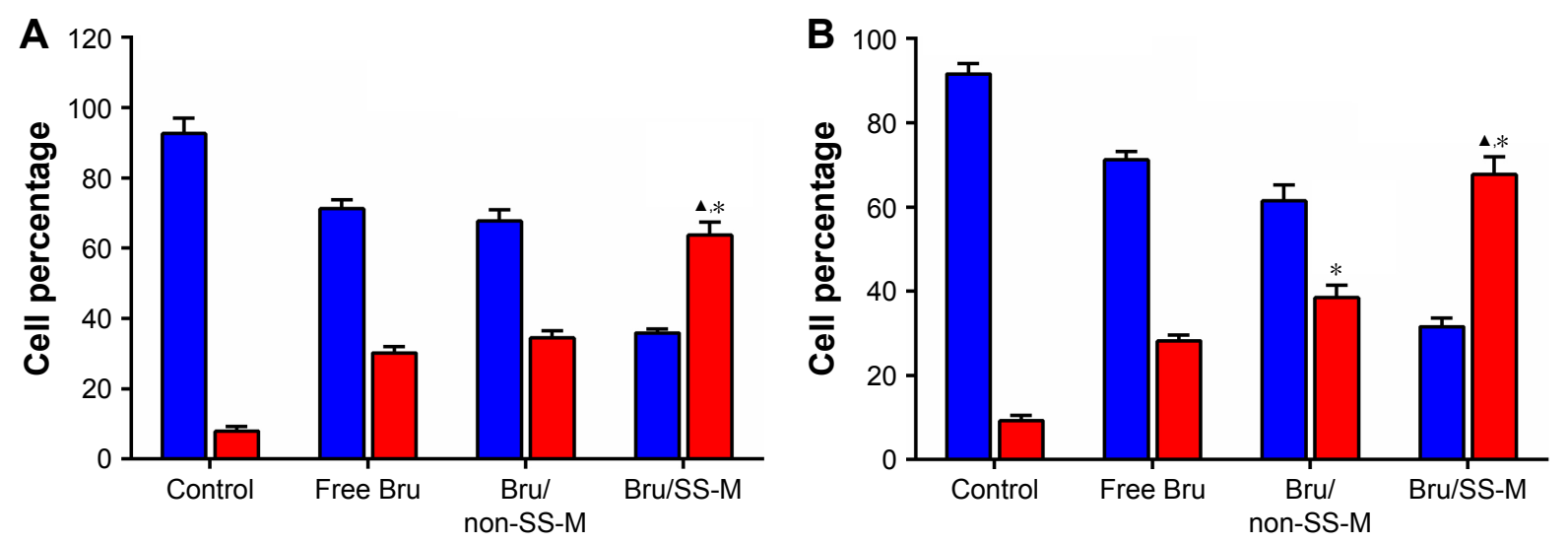

$\square$ Live cells $\square$ Apoptotic cells

C
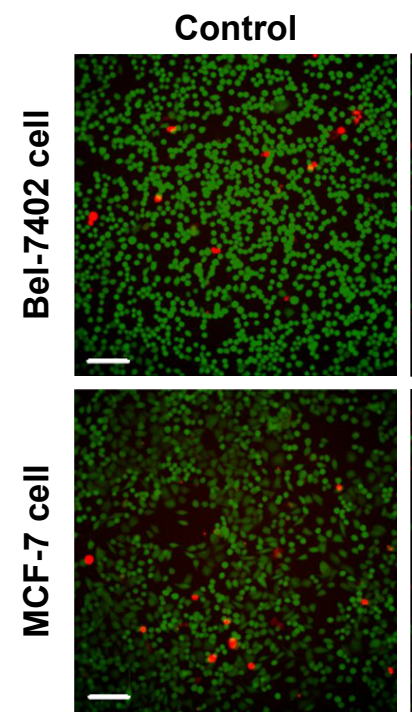

Free Bru
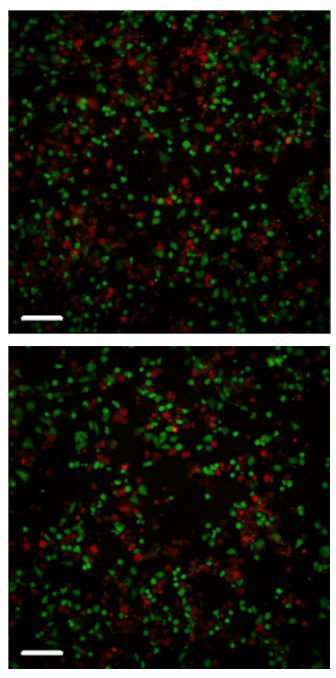

Bru/non-SS-M
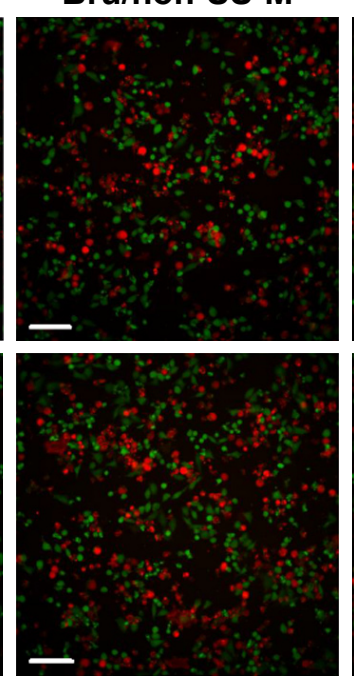

\section{Bru/SS-M}
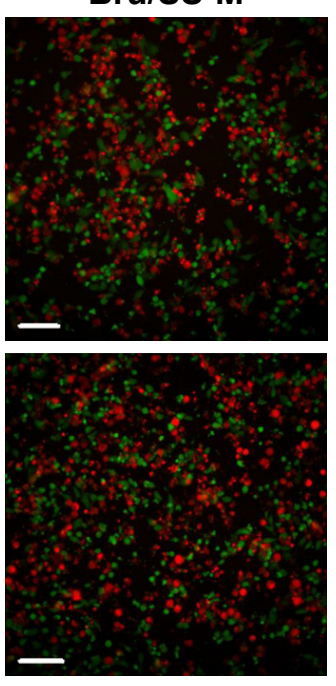

Figure 9 Bru formulations treatment caused apoptosis in cancer cells. Quantitative analysis of Bru formulations induced apoptosis by FCM in Bel-7402 (A) and MCF-7 (B) cells. (C) Cell apoptosis observed in both cell types by calcein-AM/PI double staining assay (live/dead:green/red). Notes: ${ }^{\star} p<0.05$ Bru/SS-M vs free Bru. ${ }^{*} p<0.05$ Bru/SS-M vs Bru/non-SS-M. Scale bar is $200 \mu \mathrm{m}$. Magnification $\times 20$.

Abbreviations: Bru, brusatol; FCM, flow cytometry; PI, propidium iodide.

and increased intracellular ROS have been extensively demonstrated to play pivotal roles in apoptosis. ${ }^{47}$ Therefore, we examined whether Bru loaded in micelles could induce apoptosis of cancer cells. By means of Annexin V-FITC/PI double staining, cells treated with various Bru formulations were detected by FCM. As shown in Figure 9A and B, Bru formulations resulted in increased apoptotic cell amount compared with the negative control. Particularly, significantly more apoptotic cells were observed after treatment with Bru/SS-M than after treatment with free Bru and Bru/ non-SS-M. Specifically, the apoptotic rates in Bel-7402 cells (Figure 9A) treated with free Bru and Bru/non-SS-M were $30.2 \% \pm 1.8 \%$ and $34.5 \% \pm 2.1 \%$, respectively, compared with $63.7 \% \pm 3.7 \%$ in the Bru/SS-M group. Similarly, the highest apoptotic rate in MCF-7 cells (Figure 9B) was also observed in the Bru/SS-M group.
The live/dead assay by calcein-AM/PI double staining further confirmed the pro-apoptosis activity. As shown in Figure 9C, more dead cells (red fluorescence) were observed in cells treated with Bru/SS-M. These results suggested that the inhibition effect on cell proliferation induced by Bru was at least partially attributed to its apoptotic effect, and F68-SS-LA micelles can be used as an efficient carrier for Bru to improve its anticancer effect.

\section{Conclusion}

An amphiphilic block copolymer based on Pluronic F68 and LA conjugated by redox-sensitive linkage (F68-SS-LA) was synthesized and evaluated for its potential in intracellular delivery of Bru. Due to the extended hydrophobic moiety, F68-SS-LA displayed more favorable self-assembly behavior to form flower-like micelles and higher anti-dilution 
properties than the single F68 block polymer. Bru-loaded SS micelles displayed a spherical shape, with an average size of $<100 \mathrm{~nm}$, high DL capacity, and reducible environmenttriggered drug release in vitro. A larger amount of Bru loaded in micelles than free Bru was taken into cancer cells via endocytosis. Meanwhile, to demonstrate the advantages of the redox-sensitive linkage, a nonreducible micelle structure composed of F68-LA conjugated with carbon-carbon bonds was synthesized as a control. Due to the enhanced cellular uptake and rapid drug release mediated by cleavage of disulfide bonds in a highly reductive environment, Bru/ SS-M induced higher cytotoxicity against both Bel-7402 and MCF-7 cells in a dose- and time-dependent manner than other counterparts. This enhanced anticancer effect was probably attributed to regulate the intracellular oxidation pathway, including ROS generation, mitochondria depolarization, and pro-apoptosis activity. Our findings suggested that the flexible redox-sensitive F68-SS-LA micelles exhibit great potential for Bru delivery. In future work, in vivo studies will be conducted on the pharmacokinetics, inhibition effect on the tumor volume and toxicity in mouse models.

\section{Acknowledgments}

This study was supported by the National Natural Science Foundation of China (81403120), the Macao Science and Technology Development Fund (096/2015/A3), and the Research Fund of the University of Macau (MYRG201600130-ICMS-QRCM, MYRG2015-00171-ICMS-QRCM, MYRG2016-00143-ICMS-QRCM).

The authors have no relevant affiliations or financial involvement with any organization or entity with a financial interest in or financial conflict with the subject matter or materials discussed in the manuscript.

\section{Disclosure}

The authors report no conflicts of interest in this work.

\section{References}

1. Chabner BA, Longo DL. Cancer Chemotherapy and Biotherapy: Principles and Practice. Philadelphia: Lippincott Williams \& Wilkins; 2011.

2. Hurria A, Togawa K, Mohile SG, et al. Predicting chemotherapy toxicity in older adults with cancer: a prospective multicenter study. J Clin Oncol. 2011;29(25):3457-3465.

3. Maeda H, Nakamura H, Fang J. The EPR effect for macromolecular drug delivery to solid tumors: Improvement of tumor uptake, lowering of systemic toxicity, and distinct tumor imaging in vivo. Adv Drug Deliv Rev. 2013;65(1):71-79.

4. Peer D, Karp JM, Hong S, Farokhzad OC, Margalit R, Langer R. Nanocarriers as an emerging platform for cancer therapy. Nat Nanotechnol. 2007;2(12):751-760.

5. Brannon-Peppas L, Blanchette JO. Nanoparticle and targeted systems for cancer therapy. Adv Drug Deliv Rev. 2012;64:206-212.
6. Wang AZ, Langer R, Farokhzad OC. Nanoparticle delivery of cancer drugs. Annu Rev Med. 2012;63:185-198.

7. Kwon IK, Lee SC, Han B, Park K. Analysis on the current status of targeted drug delivery to tumors. J Control Release. 2012;164(2): 108-114.

8. Mura S, Nicolas J, Couvreur P. Stimuli-responsive nanocarriers for drug delivery. Nat Materials. 2013;12(11):991-1003.

9. Zhang J, Chen R, Fang X, Chen F, Wang Y, Chen M. Nucleolin targeting AS1411 aptamer modified $\mathrm{pH}$-sensitive micelles for enhanced delivery and antitumor efficacy of paclitaxel. Nano Res. 2015;8(1): 201-218.

10. Zhang J, Zhang M, Ji J, et al. Glycyrrhetinic acid-mediated polymeric drug delivery targeting the acidic microenvironment of hepatocellular carcinoma. Pharm Res. 2015;32(10):3376-3390.

11. Stuart MAC, Huck WT, Genzer J, et al. Emerging applications of stimuli-responsive polymer materials. Nat Mater. 2010;9(2): 101-113.

12. Chen F, Zhang J, Wang L, Wang Y, Chen M. Tumor pH e-triggered charge-reversal and redox-responsive nanoparticles for docetaxel delivery in hepatocellular carcinoma treatment. Nanoscale. 2015;7(38): 15763-15779.

13. Ding J, Chen J, Li D, et al. Biocompatible reduction-responsive polypeptide micelles as nanocarriers for enhanced chemotherapy efficacy in vitro. J Mater Chem B. 2012;1(1):69-81.

14. Ding J, Shi F, Xiao C, et al. One-step preparation of reductionresponsive poly (ethylene glycol)-poly (amino acid) s nanogels as efficient intracellular drug delivery platforms. Polym Chem. 2011; 2(12):2857-2864.

15. TAWARI EP, Liu P, Wang Z, et al. Pluronic micelle-encapsulated Disufiram targets cancer stem-like cells and reverses pan-resistance in acquired resistant breast cancer cell lines. Cancer Res. 2015; 75(15 Suppl):4067.

16. Sahu A, Kasoju N, Goswami P, Bora U. Encapsulation of curcumin in Pluronic block copolymer micelles for drug delivery applications. J Biomater Appl. 2011;25(6):619-639.

17. Liu L, Yong K, Roy I, et al. Bioconjugated pluronic triblock-copolymer micelle-encapsulated quantum dots for targeted imaging of cancer: in vitro and in vivo studies. Theranostics. 2012;2(7):705-713.

18. Kabanov AV, Alakhov VY. Pluronic block copolymers in drug delivery: from micellar nanocontainers to biological response modifiers. Crit Rev Ther Drug Carrier Syst. 2002;19(1):1-72.

19. Cha M-H, Choi J, Choi BG, et al. Synthesis and characterization of novel thermo-responsive F68 block copolymers with cell-adhesive RGD peptide. J Colloid Interface Sci. 2011;360(1):78-85.

20. Zhao L, Du J, Duan Y, et al. Curcumin loaded mixed micelles composed of Pluronic P123 and F68: preparation, optimization and in vitro characterization. Colloids Surf B Biointerfaces. 2012;97: 101-108.

21. Sun CZ, Lu CT, Zhao YZ, et al. Characterization of the doxorubicinPluronic F68 conjugate micelles and their effect on doxorubicin resistant human erythroleukemic cancer cells. J Nanomed Nanotechnol. 2011;2(5):114.

22. Zhang J, Li Y, Fang X, Zhou D, Wang Y, Chen M. TPGS-g-PLGA/ Pluronic F68 mixed micelles for tanshinone IIA delivery in cancer therapy. Int J Pharm. 2014;476(1):185-198.

23. Gorrini C, Harris IS, Mak TW. Modulation of oxidative stress as an anticancer strategy. Nat Rev Drug Discov. 2013;12(12):931-947.

24. Zhao L, Li C, Zhang Y, Wen Q, Ren D. Phytochemical and biological activities of an anticancer plant medicine: Brucea javanica. Anticancer Agents Med Chem. 2014;14(3):440-458.

25. Zhao M, Lau S, Leung P, Che C-T, Lin Z. Seven quassinoids from Fructus Bruceae with cytotoxic effects on pancreatic adenocarcinoma cell lines. Phytother Res. 2011;25(12):1796-1800.

26. Ren D, Villeneuve NF, Jiang T, et al. Brusatol enhances the efficacy of chemotherapy by inhibiting the Nrf2-mediated defense mechanism. Proc Natl Acad Sci USA. 2011;108(4):1433-1438. 
27. Tang X, Wang H, Fan L, et al. Luteolin inhibits Nrf2 leading to negative regulation of the Nrf2/ARE pathway and sensitization of human lung carcinoma A549 cells to therapeutic drugs. Free Radic Biol Med. 2011; 50(11):1599-1609.

28. Ohnuma T, Matsumoto T, Itoi A, et al. Enhanced sensitivity of A549 cells to the cytotoxic action of anticancer drugs via suppression of $\mathrm{Nrf} 2$ by procyanidins from Cinnamomi Cortex extract. Biochem Biophys Res Commun. 2011;413(4):623-629.

29. Jang A, Srinivasan P, Lee NY, et al. Comparison of hypolipidemic activity of synthetic gallic acid-linoleic acid ester with mixture of gallic acid and linoleic acid, gallic acid, and linoleic acid on high-fat diet induced obesity in C57BL/6 Cr Slc mice. Chem-Biol Interact. 2008; 174(2):109-117.

30. Ibarguren M, López DJ, Escribá PV. The effect of natural and synthetic fatty acids on membrane structure, microdomain organization, cellular functions and human health. Biochim Biophys Acta. 2014; 1838(6):1518-1528.

31. Zhang W, Shi Y, Chen Y, Ye J, Sha X, Fang X. Multifunctional Pluronic P123/F127 mixed polymeric micelles loaded with paclitaxel for the treatment of multidrug resistant tumors. Biomaterials. 2011; 32(11):2894-2906.

32. Zhang J, Li Y, Gao W, Repka MA, Wang Y, Chen M. Andrographolideloaded PLGA-PEG-PLGA micelles to improve its bioavailability and anticancer efficacy. Expert Opin Drug Deliv. 2014;11(9):1367-1380.

33. Zhang W, Shi Y, Chen Y, et al. Enhanced antitumor efficacy by paclitaxel-loaded pluronic P123/F127 mixed micelles against non-small cell lung cancer based on passive tumor targeting and modulation of drug resistance. Eur J Pharm Biopharm. 2010;75(3):341-353.

34. Wen J, You KR, Lee SY, Song CH, Kim DG. Oxidative stress-mediated apoptosis the anticancer effect of the sesquiterpene lactone parthenolide. J Biol Chem. 2002;277(41):38954-38964.

35. Yang H, Li Y, Li T, et al. Multifunctional core/shell nanoparticles crosslinked polyetherimide-folic acid as efficient Notch-1 siRNA carrier for targeted killing of breast cancer. Sci Rep. 2014;4:7072.
36. Lv S, Tang Z, Zhang D, et al. Well-defined polymer-drug conjugate engineered with redox and $\mathrm{pH}$-sensitive release mechanism for efficient delivery of paclitaxel. J Control Release. 2014;194:220-227.

37. Pi F, Kaneko F, Iwahashi M, Suzuki M, Ozaki Y. Solid-state low temperature $\rightarrow$ middle temperature phase transition of linoleic acid studied by FTIR spectroscopy. J Phys Chem B. 2011;115(19): 6289-6295.

38. Albanese A, Tang PS, Chan WC. The effect of nanoparticle size, shape, and surface chemistry on biological systems. Annu Rev Biomed Eng 2012;14:1-16.

39. Batrakova EV, Han HY, Alakhov VY, Miller DW, Kabanov AV. Effects of pluronic block copolymers on drug absorption in Caco-2 cell monolayers. Pharm Res. 1998;15(6):850-855.

40. Song N, Liu W, Tu Q, Liu R, Zhang Y, Wang J. Preparation and in vitro properties of redox-responsive polymeric nanoparticles for paclitaxel delivery. Colloids Surf B Biointerfaces. 2011;87(2):454-463.

41. Ding J, Xu W, Zhang Y, et al. Self-reinforced endocytoses of smart polypeptide nanogels for "on-demand" drug delivery. J Control Release. 2013;172(2):444-455.

42. Liu X, Wang J, Xu W, et al. Glutathione-degradable drug-loaded nanogel effectively and securely suppresses hepatoma in mouse model. Int J Nanomedicine. 2014;10:6587-6602.

43. Huang K, Shi B, Xu W, et al. Reduction-responsive polypeptide nanogel delivers antitumor drug for improved efficacy and safety. Acta Biomater. 2015;27:179-193.

44. Shi B, Huang K, Ding J, et al. Intracellularly swollen polypeptide nanogel assists hepatoma chemotherapy. Theranostics. 2017;7(3):703-716.

45. Slater AF, Stefan C, Nobel I, Van Den Dobbelsteen DJ. Signaling mechanisms and oxidative stress in apoptosis. Toxicol Lett. 1995;82:149-153.

46. Laurent A, Nicco C, Chéreau C, et al. Controlling tumor growth by modulating endogenous production of reactive oxygen species. Cancer Res. 2005;65(3):948-956.

47. Reed JC. Apoptosis-based therapies. Nat Rev Drug Discov. 2002;1(2) $111-121$. 


\section{Supplementary materials Synthesis of F68-CC-LA copolymer}

The synthesis of F68-CC-LA conjugate was similar to F68SS-LA described in the manuscript (Figure S1). Briefly, based on the SA-F68-SA conjugates, 1,6-hexamethylenediamine (HMD) was used to replace cystamine (CA) and conjugated with F68-(SA) by amidation reaction in the presence of 1-(3-dimethylaminopropyl)-3-ethylcarbodiimide hydrochloride (EDC) and N-hydroxysuccinimide (NHS), in which the molar ratio was 2:1:2.5:2.5, respectively. The excess of HMD, N, N'-dicyclohexylcarbodimide (DCC), and 4-dimethylaminopyridine (DMAP) were removed by dialyzing in the dialysis tube with MWCO 3500. Subsequently, 2-fold equivalent of linoleic acid (LA) was reacted with the activated amino group in F68-(CA) ${ }_{2}$ with the aim of EDC/NHS. After stirring for $24 \mathrm{~h}$, the insoluble by-products in mixture were removed by the filterable membrane $(0.45 \mu \mathrm{m})$. F68-CC-LA copolymer containing carbon-carbon bonds was obtained by the separation of thin layer chromatography with the mixture mobile phase of methanol/chloroform $(75: 25, \mathrm{v} / \mathrm{v})$.

\section{Characterization of F68-CC-LA copolymer}

Due to the high structural similarity with F68-SS-LA, F68CC-LA displayed a similar spectrum profile. However, compared with proton nuclear magnetic resonance ( ${ }^{1} \mathrm{H}$ NMR) spectrum of F68-SS-LA copolymer in the manuscript, the methylene peaks of cystamine around $\delta 2.71-2.86 \mathrm{ppm}$ were disappeared in F68-CC-LA spectrum.

\section{In vitro cytotoxicity of copolymers}

After $48 \mathrm{~h}$ of incubation, $10-100 \mu \mathrm{g} / \mathrm{mL}$ of F68-SS-LA and F68-CC-LA copolymers did not exhibit significant cell survival inhibition. Higher than $85 \%$ of cell viability was detected, indicating the high biocompatibility of designed copolymers.

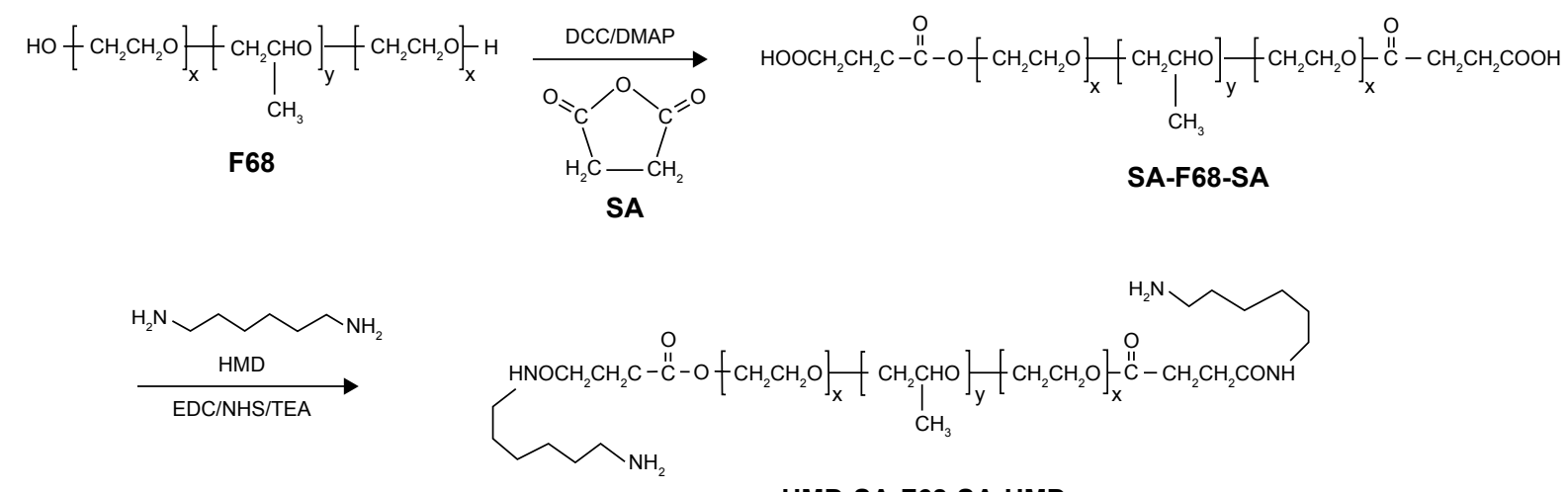

HMD-SA-F68-SA-HMD

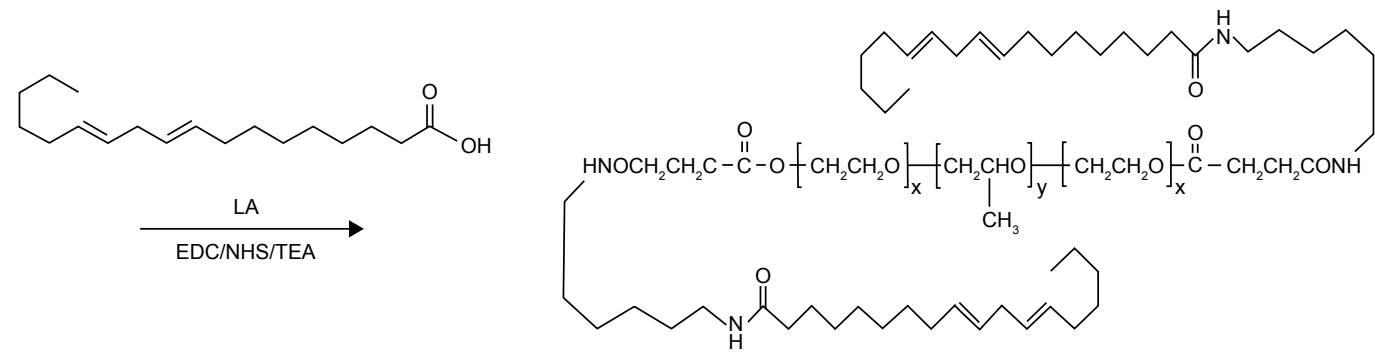

LA-HMD-SA-F68-SA-HMD-LA (F68-SS-LA)

Figure SI Synthetic route of F68-CC-LA copolymer.

Abbreviations: DCC, N, N'-dicyclohexylcarbodimide; DMAP, 4-dimethylaminopyridine; EDC, I-ethyl-3-(3-dimethylaminopropyl)-carbodiimide; HMD, I,6-hexamethylenediamine; LA, linoleic acid; NHS, N-hydroxysuccinimide; SA, succinic anhydride; TEA, triethylamine. 


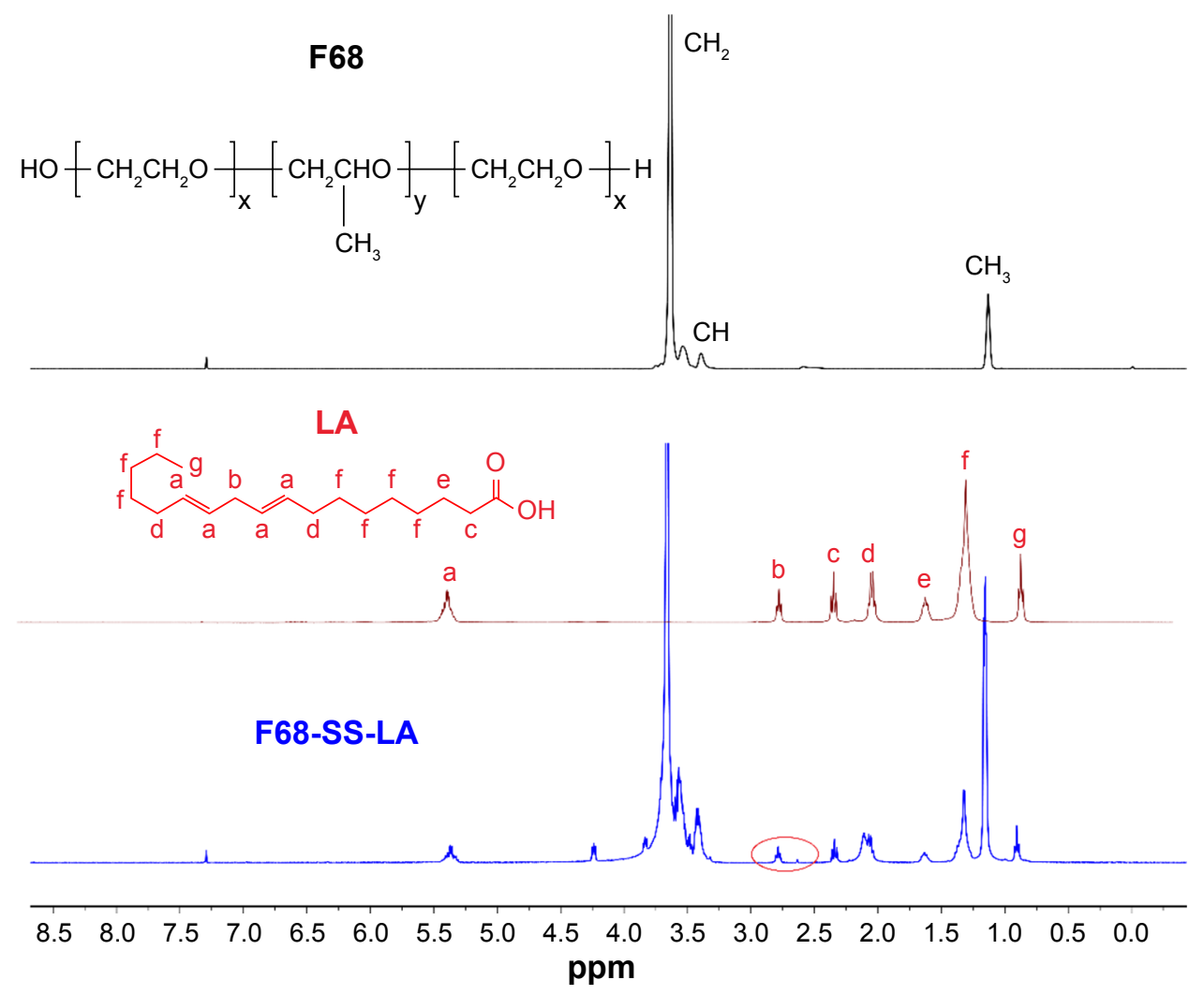

Figure S2 'H NMR spectra of F68 polymer, LA moiety, and F68-SS-LA copolymer. Abbreviations: H-NMR, proton nuclear magnetic resonance; LA, linoleic acid.

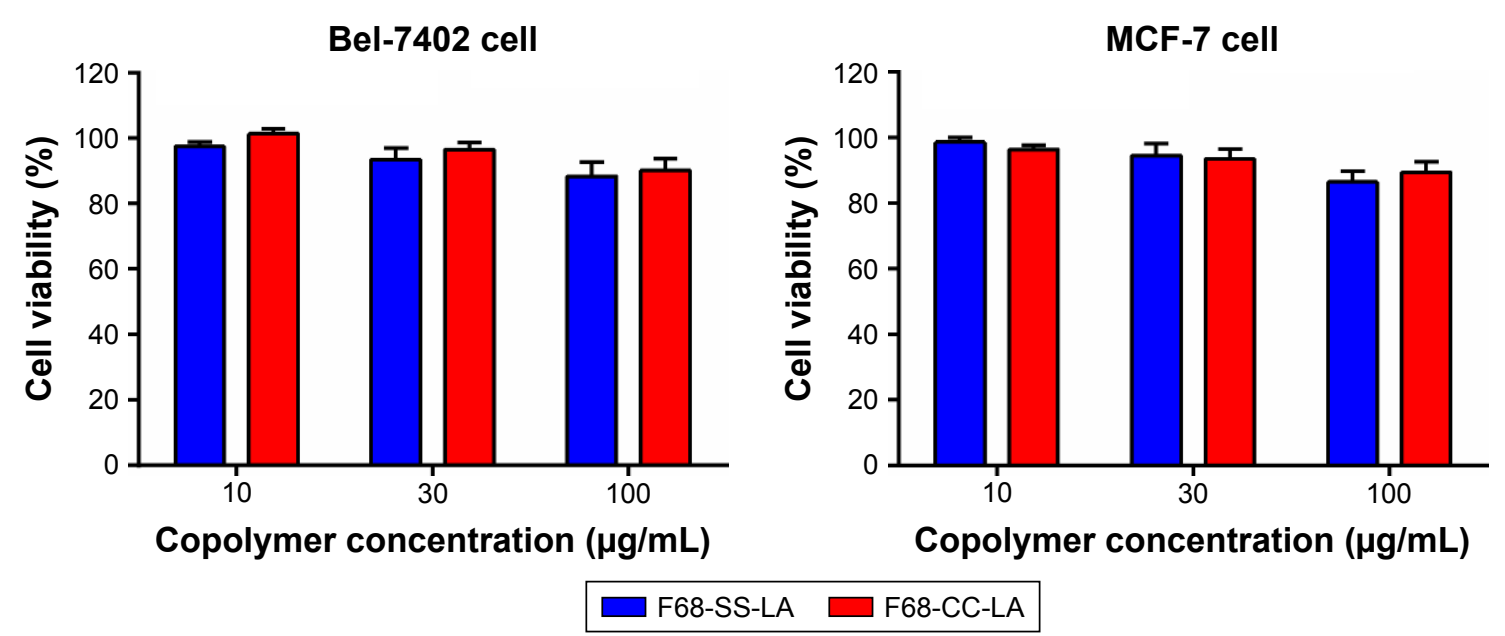

Figure S3 Cytotoxicity of F68-SS-LA and F68-CC-LA copolymer against Bel-7402 and MCF-7 cells after $48 \mathrm{~h}$ of incubation. Abbreviation: LA, linoleic acid. 


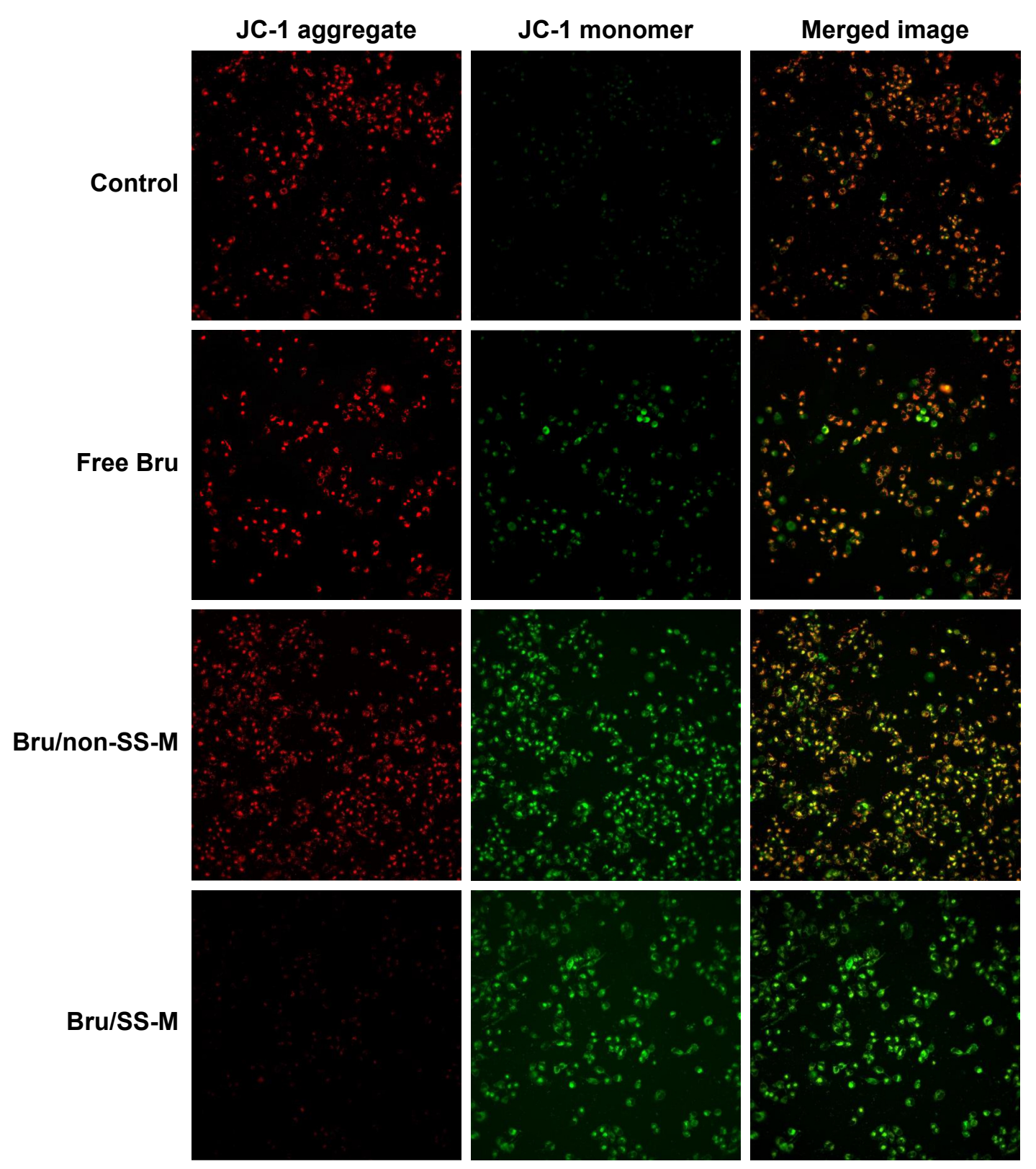

Figure S4 Fluorescence microscope images of MCF-7 cells treated with various Bru formulations (equivalent Bru concentration of $5 \mu$ M) for I 2 h treatment and subsequently stained with JC-I dye.

Notes: Bru formulations resulted in the increased green fluorescence by JC-I dye in MCF-7 cells. However, compared to free Bru and non-reducible micelles, Bru/SS-M led to the highest amount of green fluorescence, suggesting effect of MMP significantly decreased and with great mitochondrial disturbance activity. Scale bar is $200 \mu \mathrm{m}$. Magnification $\times 200$.

Abbreviation: Bru, brusatol.

International Journal of Nanomedicine

\section{Publish your work in this journal}

The International Journal of Nanomedicine is an international, peerreviewed journal focusing on the application of nanotechnology in diagnostics, therapeutics, and drug delivery systems throughout the biomedical field. This journal is indexed on PubMed Central, MedLine, CAS, SciSearch ${ }^{\circledR}$, Current Contents $₫ /$ Clinical Medicine,

\section{Dovepress}

Journal Citation Reports/Science Edition, EMBase, Scopus and the Elsevier Bibliographic databases. The manuscript management system is completely online and includes a very quick and fair peer-review system, which is all easy to use. Visit http://www.dovepress.com/ testimonials.php to read real quotes from published authors. 